\title{
Entropy Contributions to Transition State Modelling
}

Authors: Kristof De Wispelaere, Louis Vanduyfhuys and Veronique Van Speybroeck

Center for Molecular Modeling, Ghent University, Technologiepark 903, B-9052 Zwijnaarde, Belgium

\section{Introduction}

Entropy plays a major role in many physical and chemical phenomena. This is certainly also the case for reactions taking place on surfaces and for catalysis taking place in confinement such as zeolites or Metal-Organic Frameworks (MOFs). The role of entropy has certainly not been underestimated but the exact quantification both from experimental and theoretical points of view has proven to be extremely challenging. Recently Campbell and Sellers succeeded in quantifying entropies of adsorbed molecules on crystalline materials by analyzing numerous measurements of equilibrium adsorption isotherms and thermal desorption data.[1] Their results show that entropies of adsorbed species are much larger than originally thought and correlate linearly with the entropies of gaseous molecules.[2]

Also within the field of material's science, major progress has been made to understand flexibility of framework materials and temperature-driven transitions. Recently Butler et al. wrote a review on the important role of entropy in formation and defect engineering of hybrid inorganic-organic systems.[3] Many application relevant properties are closely related to entropic effects, such as sorption properties, temperature driven phase transitions, responses to external pressures, thermal conductivities etc. For the MOF material MIL-53, for example, a recent theoretical and 
experimental study unraveled its pressure induced phase transitions in which entropy plays a crucial role, illustrating its potential use as a shock absorber.[4] Also transitions between the COK14 and -COK-14 form of the recently synthesized OKO-structured zeolite were found to entropy driven.[5]

Within this perspective we focus on the role of entropy for reaction mechanisms and kinetics taking place in nanoporous materials. Most of the examples are taken from zeolite catalyzed reactions, but the methods are also applied in other fields such as reactions taking place in new emerging materials with promising properties such as Metal-Organic Frameworks. This perspective is not intended to be comprehensive, but rather highlights some interesting evolutions with respect to entropy contributions to modeling reactions taking place in nanoporous materials. The field of entropy in various domains is very broad and the interested reader is referred to dedicated reviews on the topic.[3, 6-9] Furthermore, no comprehensive overview of all theoretical aspects related to entropy determination will be given. We only highlight those aspects that made their entrance within the field of zeolite catalyzed reactions or which have the potential to become very important in the near future.

Adsorption and activation of molecules in zeolites or MOFs are subject to the confined space of the nanoporous host material. For such systems, a delicate balance between enthalpic and entropic effects (vide infra) often leads to very complex reaction kinetics. Furthermore, there is experimental evidence that the preferred reaction mechanism may be critically dependent on the operating conditions. One example concerns methylation reactions taking place in Brønsted acidic zeolites, which can either occur via a concerted or stepwise mechanism. Iglesia and co-workers gave experimental and theoretical evidence that a stepwise methanol dehydration mechanism would be preferred at higher temperatures and low pressures because it involves smaller transition 
states with higher enthalpy and entropy, than those in the concerted route.[10] Similar conclusions have been drawn by Brogaard et al. based on a combined theory-experiment approach including micro-kinetic modeling.[11] From a modeling perspective this also imposed major challenges as it becomes necessary to evaluate the reactions of interest at realistic experimental conditions, such as temperature, pressure, realistic feedstocks,...[12-14] It is only recently that advanced methods taking into account this type of complexity are entering the scene. [12-23] So far, most theoretical procedures for determining the reaction mechanisms and reaction rates rely on so-called static methods where only a limited number of points on the potential energy surface are taken into account. Even such approach is far from straightforward as the systems of interest typically have many degrees of freedom. Therefore, mapping the potential energy surface - even at $0 \mathrm{~K}$ - with ab initio methods such as density functional theory (DFT) or quantum chemical wavefunction methods is a challenge on its own. This, however, gives only a limited insight as temperature effects, in particular entropy, often play decisive roles in many physical or chemical conversions. The aim of a molecular modeling study is often to calculate accurate reaction kinetics of elementary steps. In this respect, one often calculates free energy barriers, which are related with rate coefficients via the Arrhenius law. Especially entropic contributions are of paramount importance in zeolite catalysis, since many zeolite-catalyzed reactions occur at high temperature. Nonetheless, entropic effects are not well understood as they are difficult to measure or calculate. Apart from effects typically related to operating conditions, confinement also plays a decisive role in zeolite catalysis.[6, 24-34] In general, a more confined space provides a better stabilization of intermediates and/or transition states, but often also leads to more loss of entropy.[20, 35] Such effects have recently been reported for protolytic propane cracking in mordenite [20] and for alkene methylation in different zeolite topologies.[35] On the other hand, for zeolite chemistry in 
large pore materials, configurational freedom rather than confinement plays an important role and should be accounted for in modeling studies. For benzene methylation in the large-pore AFI materials, many isoenergetic reactant and transition states were found, $[12,13,36]$ making free energy and entropy calculations based on static optimizations at $0 \mathrm{~K}$ a less attractive technique (see section 5 of this perspective). In this case not one single transition state determines the overall reaction kinetics but one needs to account for larger portions of phase space. For the reaction at hand taking place in the large pore zeolite with AFI topology, advanced molecular dynamics (MD) simulations were used to take the degeneracy of the different states appropriately into account. As such free energies at operating conditions were found. However, it remains challenging to extract an accurate entropic contribution from free energy calculations from advanced MD simulations (see section 5).

Furthermore, for heterogeneously catalyzed reactions it has been reported that there might exist a subtle entanglement of the enthalpy and entropy change during the reaction.[37-41] This effect has often been referred to as the "compensation effect" in literature and has been the subject of many debates so far.[38, 42] A schematic representation of the compensation effect on the overall kinetics is shown in Figure 1, assuming that the intrinsic free energy of activation remains constant. A typical example that was discussed in literature involves alkane cracking, where it was shown that the true activation energy is virtually invariant with the alkane chain length, whereas the apparent activation energy was rapidly decreasing with longer alkanes.[43] With longer chain length the enthalpy of adsorption is stronger due to additional Van der Waals interactions of the extra methylene groups, but simultaneously such stronger adsorption leads to more loss of entropy as fewer conformations are possible compared to the gas phase molecules. Theoretical evidence for the compensation effect was given by Smit et al. who used Monte Carlo simulations to estimate 
the free energies of adsorption.[44] Experimentally, Van Santen and co-workers were able to explicitly determine the entropic and enthalpic effects for $\mathrm{n}$-hexane hydroisomerization on a variety of zeolites and could also clearly observe a compensation effect.[45] These lively discussions emphasize the importance to track both enthalpy and entropy contributions to each step of the reaction path.

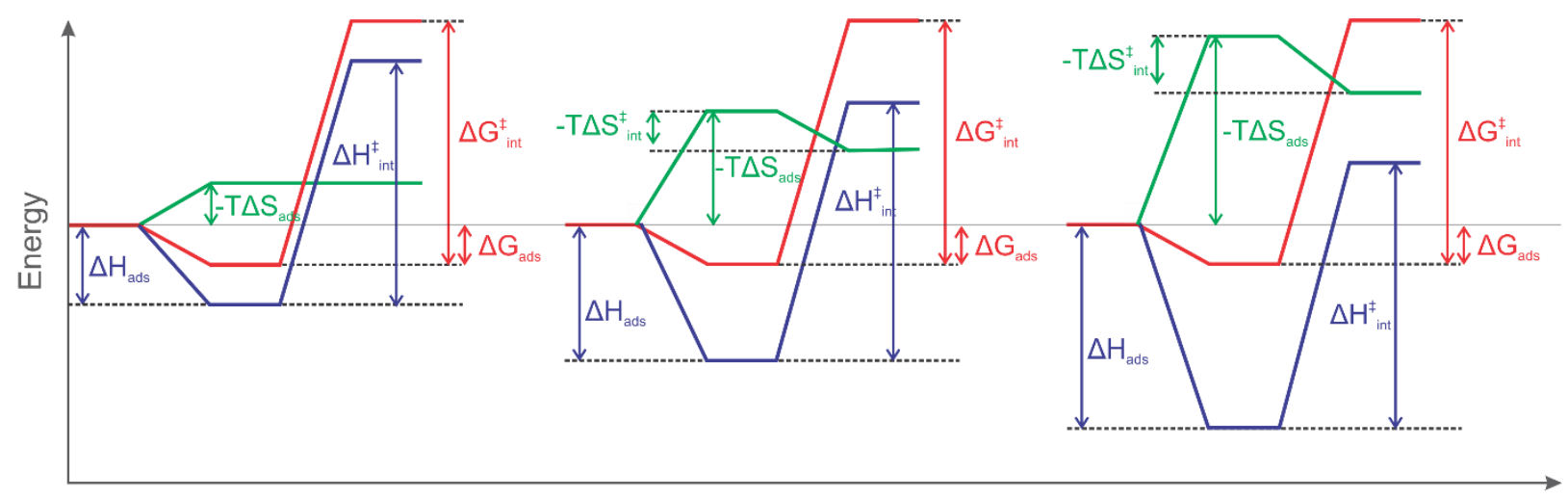

Reaction coordinate

Figure 1. Schematic illustration of the compensation effect as the adsorption enthalpy $\left(\Delta \mathrm{H}_{\mathrm{ads}}\right)$ increases, whereas the intrinsic standard enthalpy of activation $\left(\Delta \mathrm{H}^{\dagger}\right.$ int $)$ and the free energy of activation $\left(\Delta \mathrm{G}_{\text {int }}^{\dot{1}}\right)$ are held constant. Also, the evolution of the entropy of adsorption $\left(-\mathrm{T} \Delta \mathrm{S}_{\mathrm{ads}}\right)$ and activation (-T $\left.\Delta \mathrm{S}_{\text {int }}^{\dagger}\right)$ are shown. Free energy, enthalpy and entropy are shown in red, blue and green respectively. Apparent values, i.e. those that are measured, are not indicated.

Futhermore, when micro-kinetic models are used, accurate enthalpy and entropy barriers for each reaction step are also required.[11, 46] These arguments underline the necessity of an accurate determination of entropic contributions when modeling catalytic reactions at operating conditions.

In the last decade, major advances have been made to estimate the reaction kinetics from theory. [47] Seminal papers appeared by Sauer and co-workers to estimate the reaction enthalpies of zeolite catalyzed reactions with chemical accuracy.[48-50] Yet it is only recently that new 
ingenious methods are also applied to estimate the entropy contributions to the reaction kinetics with greater accuracy. Within this perspective, we will give an overview of the current status in this field and try to pinpoint further challenges and opportunities from a modeling viewpoint.

\section{Comparison between experimental and theoretical rate constants}

Transition state theory is without doubt the most popular method to estimate reaction kinetics. Within this concept, each successful reaction path should pass through a transition state or activated complex. Due to these drastic approximations, the reaction kinetics may be described by calculating a limited number of points on the potential energy surface. A schematic illustration for a typical zeolite catalyzed reaction is given in Figure 2.

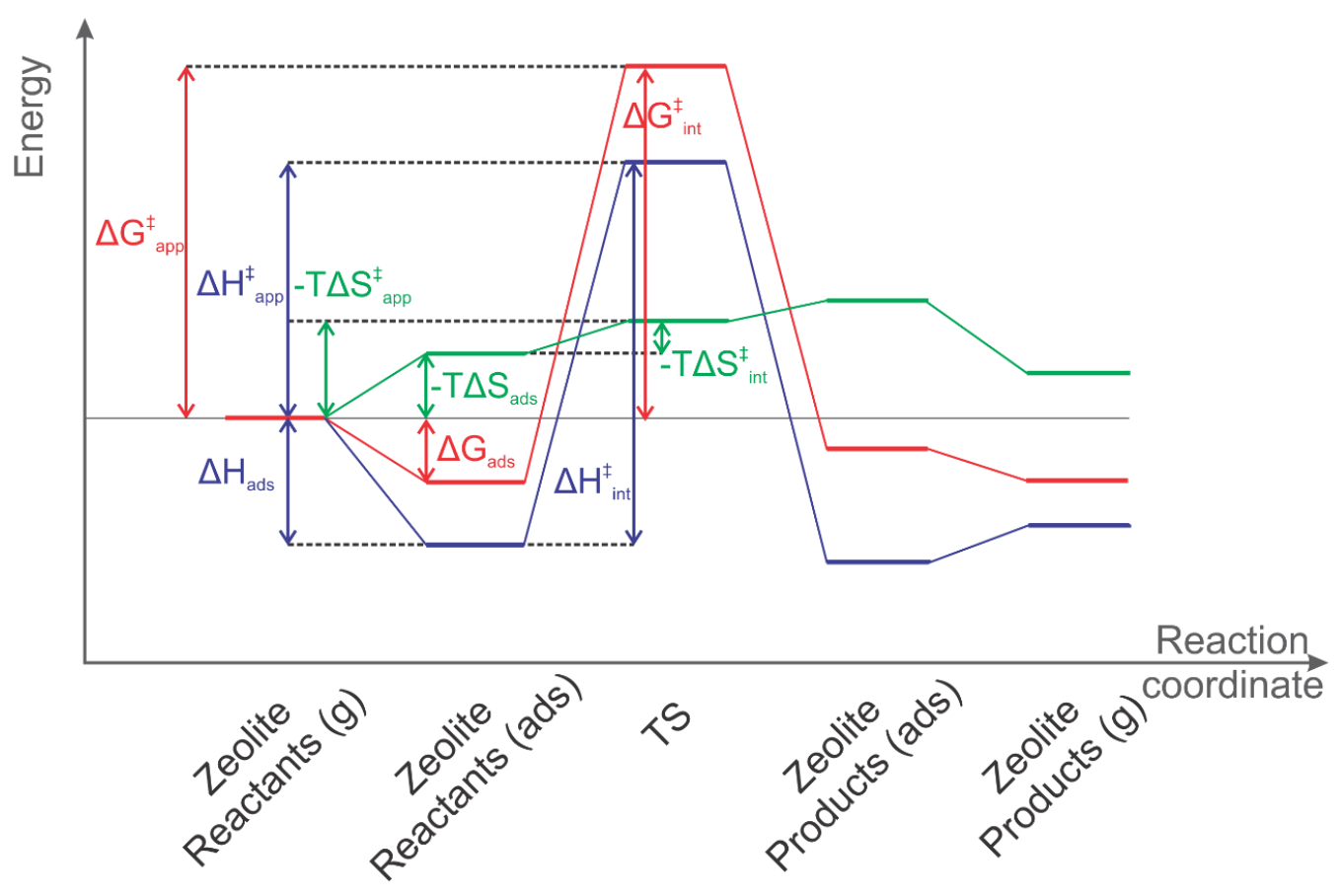

Figure 2. Schematic illustration of the enthalpy (blue curve), entropy (green curve) and free energy (red curve) profile for a zeolite-catalyzed reaction. $\Delta \mathrm{H}_{\text {app }}^{\ddagger},-\mathrm{T} \Delta \mathrm{S}_{\text {app }}^{\ddagger}$ and $\Delta \mathrm{G}^{\ddagger}$ app are the enthalpy, 
entropy and free energy barriers; $\Delta \mathrm{H}^{\dagger}$ int,$-\mathrm{T} \Delta \mathrm{S}^{\ddagger}$ int and $\Delta \mathrm{G}^{\ddagger}$ int are the intrinsic enthalpy, entropy and free energy barriers.

The reaction kinetics is then evaluated by localizing stable minima on the $0 \mathrm{~K}$ potential energy surface for each of the points along the reactions coordinate, including adsorption and reaction steps. To estimate all thermodynamic functions, the partition function needs to be evaluated. In such approach, the internal motions of the system are approximated as a superposition of a series of independent harmonic oscillators, characterized by their eigenfrequencies or normal modes. This procedure is also often referred to as a normal mode analysis (NMA).[51, 52] The reaction rate constant within standard TST is given by the following expression:

$k(T)=\frac{k_{B} T}{h} V^{-\Delta^{\ddagger} n} e^{-\frac{\Delta^{\ddagger} G}{R T}}=\frac{k_{B} T}{h} V^{-\Delta^{\ddagger} n} e^{\frac{\Delta^{\ddagger} S}{R}} e^{-\frac{\Delta^{\ddagger} H}{R T}}$

In this expression $\mathrm{k}_{\mathrm{B}}$ represents the Boltzmann constant, $\mathrm{T}$ stands for the temperature, $\mathrm{V}$ is the volume, $\mathrm{h}$ is Planck's constant, $\mathrm{R}$ is the universal gas constant and $\Delta^{\ddagger} n$ is the molecularity of the reaction. The entropy contributions of the reaction are thus captured in the pre-exponential factor of the reaction.

When studying heterogeneous catalyzed reactions, attention was mostly given to an accurate determination of the enthalpy contributions. During the last decade some studies also pointed to the importance of the pre-exponential factor. To directly compare theoretical rate constants with experimental data, a proper estimate of the heat of adsorption of the reactants and also surface coverages are needed.[38, 53] 
A reaction that received a lot of attention to estimate accurate reaction kinetics is the methylation reaction of alkenes with methanol in ZSM-5 (Scheme 1) Svelle and co-workers succeeded to estimate the methylation rate of alkenes (ethene, propene and n-butene) without interference of side reactions, by utilizing a reaction system consisting of ${ }^{13} \mathrm{C}$ methanol and ${ }^{12} \mathrm{C}$ alkene, and choosing the conditions such that secondary reactions are inhibited.[54, 55]

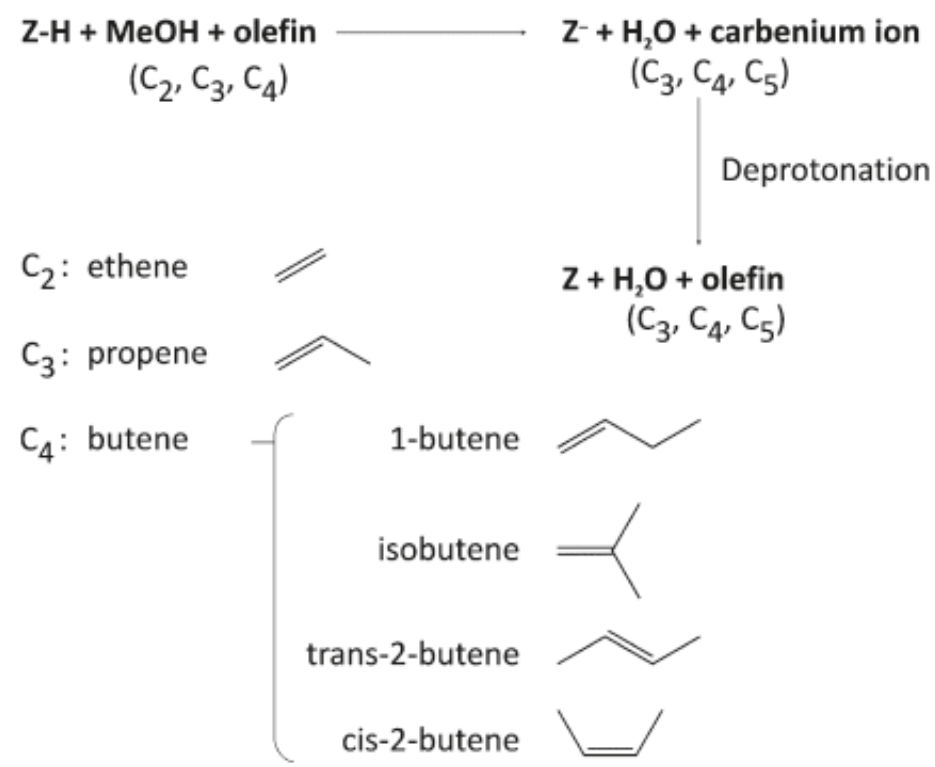

Scheme 1. Methylation of alkenes with methanol. Taken from ref. [56]

Seminal theoretical work was performed by Sauer and co-workers to estimate enthalpy barriers for these reactions within chemical accuracy (within $4 \mathrm{~kJ} / \mathrm{mol}$ compared to experimental data). The authors used a multistep MP2:DFT approach that was originally introduced by Tuma and Sauer. $[49,50]$ and that succeeds in producing approximate MP2 energy estimates of the periodic system.[48] A summary of the enthalpy barriers found in that work is shown in Figure 3. The paper of Svelle et al. may be regarded as a landmark paper since it proved for the first time that enthalpy barriers for zeolite catalyzed reactions could be obtained with near chemical accuracy. The approach is however also computationally very demanding and is certainly not feasible as a routine 
procedure. This study underlines the fact that initially attention was primarily focused on obtaining very accurate enthalpies of the reactions.

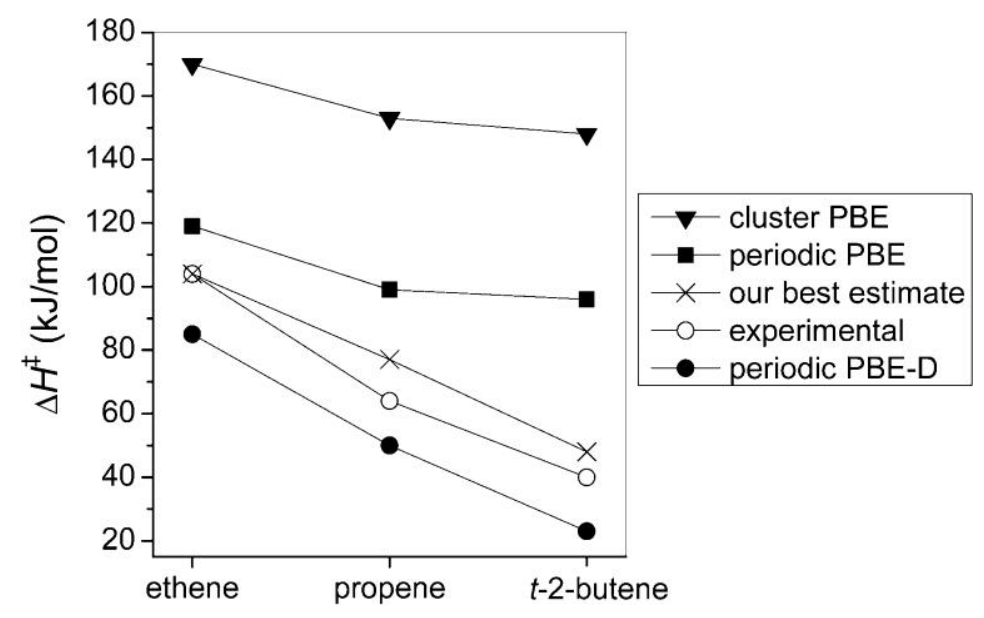

Figure 3. Apparent enthalpy barriers for the methylation of alkenes in H-ZSM-5 obtained with various computational schemes as compared to experimental data. Results indicated with (x) correspond to the hybrid MP2:DFT-based scheme. [Reprinted with permission from [48], Copyright 2009 American Chemical Society]

Later on, some of us reevaluated the same reaction, using a much cheaper computational protocol.[56] We adopted a very efficient ONIOM based scheme on extended finite zeolite clusters. Excellent agreement was found for the apparent barriers and furthermore we also derived reaction rates that were directly comparable to experimental data for the first time. The comparison between theoretical and experimental rate constants is shown in Figure 4. The agreement is very good, as the theoretical predictions deviate by no more than a factor 10 compared to the experimental rate constants. At that time, we coined the term kinetic accuracy for rate constants that deviate no more than one order of magnitude compared to their benchmark values. 


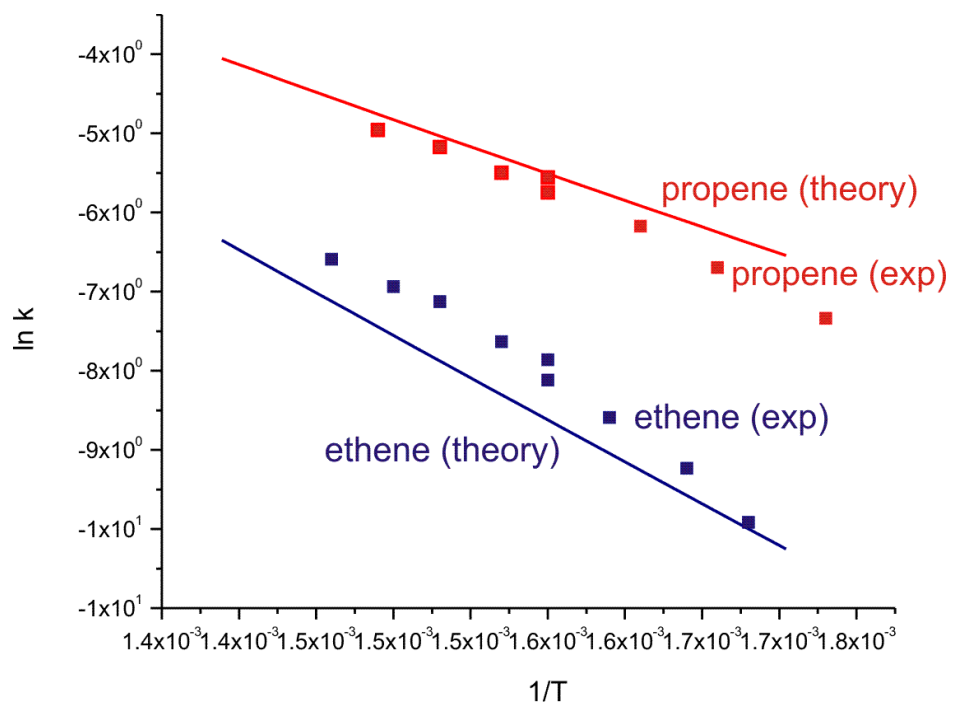

Figure 4. Theoretically and experimentally determined Arrhenius plots for the methylation of ethene and propene with methanol. [Reprinted with permission from ref.[56], Copyright 2011 American Chemical Society)

Despite these early successes of transition state theory within the field of zeolite chemistry, some caution is warranted to generalize the conclusions made here. Transition state theory as applied here relies on the harmonic oscillator approximation where all normal modes are described as harmonic oscillators. This is a serious simplification especially for low frequency modes or socalled soft vibrational modes. In this case the PES along these coordinates is very flat and the motion can no longer be approximated by a harmonic oscillator. Typical soft modes correspond to weakly bound complexes in the pores of the zeolite or lattice motions. Furthermore, these lowest vibrational motions give the largest contribution to the entropy of the system. First of all, it has been found notoriously difficult to converge structures with many degrees of freedom to such extent that all frequencies are positive. Second even when this goal is achieved, the numerical noise on the determined frequencies is large. As the lowest vibrational modes contribute the most to the vibrational entropy, large errors are introduced using such approach. This problem has been 
acknowledged and various techniques have been proposed to circumvent this problem, which vary from simple numerical tricks to prevent unreasonable contributions to the entropy to more ingenious algorithms which are physically founded. An overview of various techniques to treat these soft modes is given in the next section.

\section{Beyond the harmonic oscillator approximation in a static approach}

The vibrational entropy associated with a harmonic oscillator frequency of $\omega$ is given by the following expression:

$$
S_{V}=R\left[\frac{\hbar \omega}{k T\left(e^{\hbar \omega / k_{B} T}-1\right)}-\ln \left(1-e^{-\hbar \omega / k_{B} T}\right)\right]
$$

Where $\mathrm{k}_{\mathrm{B}}$ represents the Boltzmann constant, $\mathrm{T}$ stands for the temperature, $\mathrm{h}$ is Planck's constant and $\mathrm{R}$ is the universal gas constant. This expression diverges for frequencies approaching zero.

In many papers some of the low vibrational frequencies are simply replaced by an artificial low frequency, for example $25 \mathrm{~cm}^{-1}$ or another arbitrary low frequency value. This method simply avoids the divergence of the harmonic oscillator approach for frequencies going to zero, but has no physical motivation. Furthermore, in many cases it has been used to remove some of the difficult negative frequencies artificially from the frequency spectrum. As an example we mention the work of De Moor et al. in which spurious imaginary frequencies, corresponding to a translation or rotation of hydrocarbon molecule inside a zeolite pore, are replaced by a normal mode of 50 $\mathrm{cm}^{-1} \cdot[57]$ 
In 2012, Grimme proposed a completely black-box type procedure to remedy the problem of the low frequencies.[58] Instead of using expression (2) for the entropy, he proposed to use following expression:

$S=w(\omega) S_{V}+[1-w(\omega)] S_{R}$

$w(\omega)=\frac{1}{1+\left(\omega_{0} / \omega\right)^{a}}$

Which is an interpolation of the rotational $\left(\mathrm{S}_{\mathrm{R}}\right)$ and harmonic vibrational $\left(\mathrm{S}_{\mathrm{V}}\right)$ entropy, with $\mathrm{w}(\omega)$ given by the Head-Gordon damping function.[59] For frequencies $\omega$ lower than the chosen cut-off value of $\omega_{0}$ the vibrational entropy is replaced by the corresponding free-rotor entropy. In the original study of Grimme, $\omega_{0}$ was chosen as $100 \mathrm{~cm}^{-1}$ although it was shown that the computed entropies were not very sensitive to the particular choice of the cut-off frequency as far it was chosen reasonable (between 50-150 $\mathrm{cm}^{-1}$ ). Similar procedures and cut-off values were also used by other researchers.[60, 61] The proposed procedure is an easy method for practical applications, although it has no real physical foundation. This technique allows a smooth interpolation between a free rotor at lower frequencies and a harmonic oscillator at higher frequencies using a frequencydependent weighting function. It has been applied numerously, also in heterogeneous catalysis. For example, Janda et al. applied the procedure successfully in a study on the n-alkane monomolecular cracking in ZSM-5.[62] They found good agreement between the internal entropy of activation using a composite hindered rotor approach adapted from Grimme's original formulation to account also for internal rotations.

A theoretically more founded procedure was proposed by some of us and is referred to as the mobile adsorbate method approach. In this approach some of the low lying frequencies are not treated in the $\mathrm{HO}$ limit but rather as free translational or rotational motions of the adsorbate in the 
zeolite pores.[63, 64] The method was first introduced and tested to calculate accurate adsorption entropies of physisorption of n-octane and isobutene and chemisorption of t-butyl carbenium ion, i-butoxy and t-butoxy in H-ZSM-22 and H-FAU. Some of the complexes correspond to loosely bound complexes in the pores of the zeolite where no chemical bond exists between the adsorbate and the host framework. Figure 5 shows some relevant physisorbed and chemisorbed species in H-ZSM-22 and H-FAU.
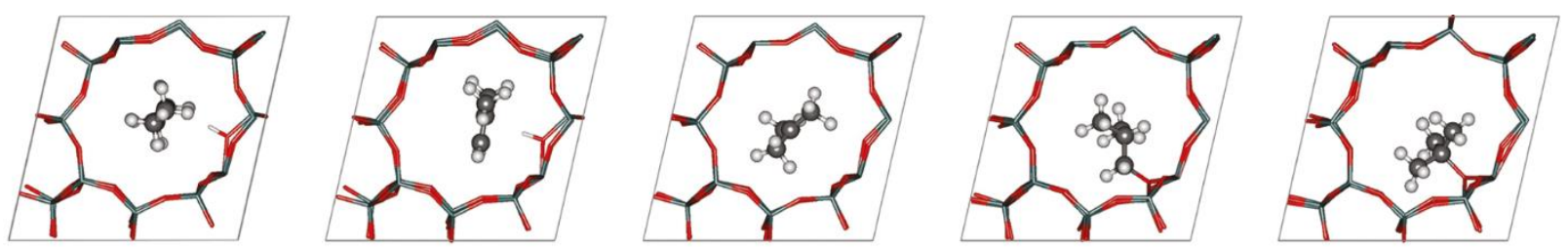

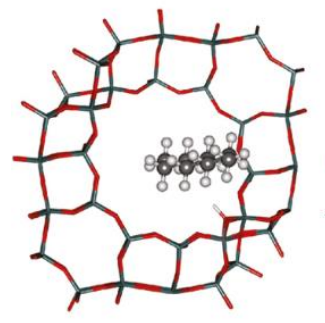

n-octane

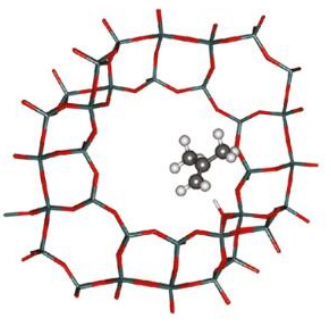

isobutene

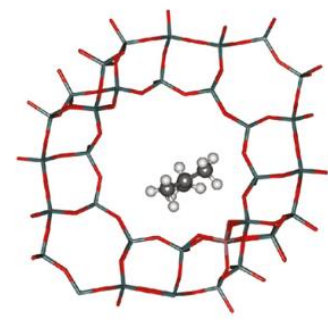

t-butyl

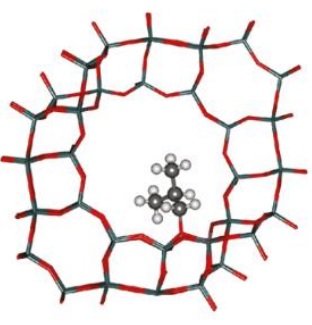

i-butoxy

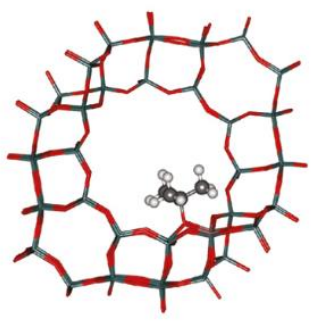

t-butoxy

Figure 5. Physisorption and/or chemisorption complexes of n-octane and isobutene in H-ZSM-22 and H-FAU (top and bottom figures, respectively). Taken from ref. [63]

When using the harmonic oscillator approximation for those loosely bound complexes, the entropy losses associated with adsorption are seriously overestimated, since the adsorbate still has significant rotational and translational degrees of freedom in the pores of the material. The standard HO approximation is referred in this context as the immobile adsorbate method since the adsorbed molecule is completely immobilized on the zeolite surface. In the proposed mobile adsorbate method, some of the low vibrational modes originating from translational and/or rotational contributions are removed from the total partition functions and are substituted in the 
partition function by free translational and/or rotational contributions. The selection of these frequencies is performed in an unambiguous way based on a mobile block Hessian (MBH) partial Hessian vibrational analysis (PHVA).[64-66] These procedures were performed using an in-house developed software program TAMKIN, a post-processing toolkit for normal mode analysis, thermochemistry and reaction kinetics.[67] Figure 6 illustrates the 2D free translation and the 1D free rotation of the loosely bonded complexes in H-ZSM-22.
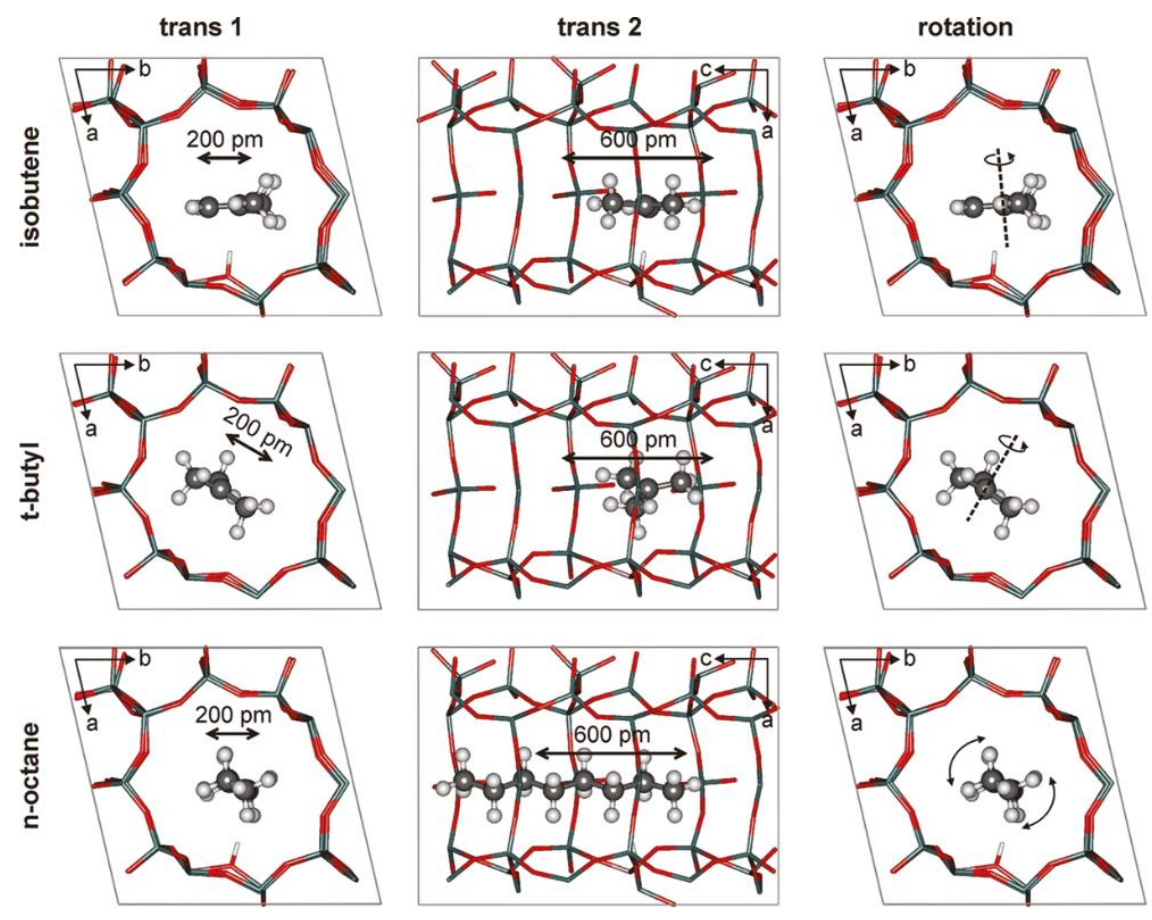

Figure 6. Impression of the 2D free translation and the 1D free rotation of the isobutene, t-butyl, and n-octane in H-ZSM-22. Taken from ref. [63]

Using such MBH approach, the physisorption entropies were in much better agreement with experimental values. Table 1 compares the physisorption energies of n-octane in H-ZSM-22 determined using the immobile and mobile adsorption method with data from experiments performed by Denayer et al. $[68,69]$ The immobile adsorbate method seriously overestimates the adsorption entropies by about $50 \mathrm{~J} / \mathrm{mol} / \mathrm{K}$. The mobile adsorbate method brings the theoretical 
predictions much closer to the experimental values and in general the calculated entropies differ by no more than $10-15 \mathrm{~J} / \mathrm{mol} / \mathrm{K}$ compared to other reference data from literature.

Table 1. Comparison of calculated values for the physisorption entropy of n-octane with the immobile and mobile adsorbate approach [26, 63] and experimentally measured values [68, 69].

\section{Physisorption entropy [J/mol/K]}

\begin{tabular}{c|c|c}
\hline Immobile adsorbate [26, 63] & Mobile adsorbate [26, 63] & $\begin{array}{c}\text { Experimental values of } \\
\text { Denayer [68, 69] }\end{array}$ \\
\hline-189 & -148 & -159 \\
\hline
\end{tabular}

More recently, Piccini and Sauer proposed a suite of procedures to remedy various problems associated with the low frequency modes. First of all, a computational strategy is proposed that improves the numerical accuracy of the frequency calculations and second an approximative scheme was proposed to account for anharmonicity in the vibrational entropy for each individual vibrational mode.[70] Instead of optimizing structures in cartesian coordinates, a re-optimization step is proposed in normal mode coordinates. Optimizations using cartesian coordinates do often not succeed in finding minima for floppy structures even with tight optimization procedures. Second, the Hessian is also numerically evaluated in normal coordinates to reduce the numerical noise on the obtained frequencies. Once stable frequencies have been obtained, diagonal anharmonic corrections are computed using a procedure proposed by Beste et al.[71] An anharmonic correction is proposed using a model potential that is fitted to the PES along each normal mode independently, thereby neglecting mode-mode coupling. To this end a Schrödinger 
equation is solved for all modes independently in a basis of harmonic functions with a fourth or sixth order model potential. The Hamiltonian of the $\mathrm{k}^{\text {th }}$ mode has the following expression:

$\mathcal{H}=-\frac{\hbar^{2}}{2} \frac{d^{2}}{d Q_{k}^{2}}+a_{0}+a_{1} Q_{k}+a_{2} Q_{k}^{2}+a_{3} Q_{k}^{3}+a_{4} Q_{k}^{4}+a_{5} Q_{k}^{5}+a_{6} Q_{k}^{6}$

With $\mathrm{Q}_{\mathrm{k}}$ the normal coordinate of the mode and $\mathrm{a}_{\mathrm{i}}(\mathrm{i}=0, \ldots, 6)$ the polynomial fitting coefficients of the potential. Finally, by diagonalization of the Hamiltonian matrix in a certain number of harmonic basis functions, a quantum anharmonic vibrational partition function is calculated until it converges with numerical accuracy. In a series of later papers, they further refined the method to solve the Schrödinger equation for potentials that were obtained by curvilinear distortions of the normal modes rather than using rectilinear Cartesian distortions. It was shown that this procedure allowed to minimize anharmonic couplings between the one-dimensional oscillators.[72-75] The new routines were implemented in a series of codes that interface with the popular VASP program for solid state calculations.[76-79] The methods were extensively tested for adsorption of small alkane molecules in H-Chabazite.[80] Together with the group of Lercher, new experimental data were also determined for the heats of adsorption of methane, ethane and propane.

Figure 7 shows the calculated thermodynamic functions obtained from experiment and various theoretical models. The separate contributions to the enthalpy and entropy are shown in Figure 7a. The effect of anharmonicity has the largest impact on the entropy of adsorption and the corrections are substantial. Corrections of the order of $15-20 \mathrm{~kJ} / \mathrm{mol}$ are obtained for the $\mathrm{T} \Delta \mathrm{S}$ term. Similar effects are observed for the direction of the entropy changes, entropy losses are overestimated in the $\mathrm{HO}$ model and thus less stable adsorption is predicted compared to experimental values. Figure $7 \mathrm{~b}$ also compares the free energy results of Piccini with some simpler 
adsorption models proposed by Tait et al. [81] Following models are included in Figure 7b: the immobile adsorbate model where no translations or rotations are allowed in the adsorbed state, a rotating adsorbate where rotation along the principal axes of inertia are allowed in the adsorbed state and a mobile adsorbate where both 2D translations and a specific number of rotations are taken into account. The mobile adsorption model gives much better predictions compared to the experimental values and are in much closer agreement with the anharmonic corrections predicted by Piccini and Sauer. The model proposed by Piccini et al. gives yet very systematic anharmonic corrections compared to the experimental thermodynamic data and displays the expected decrease in adsorption free energy along the series of methane, ethane and propane.
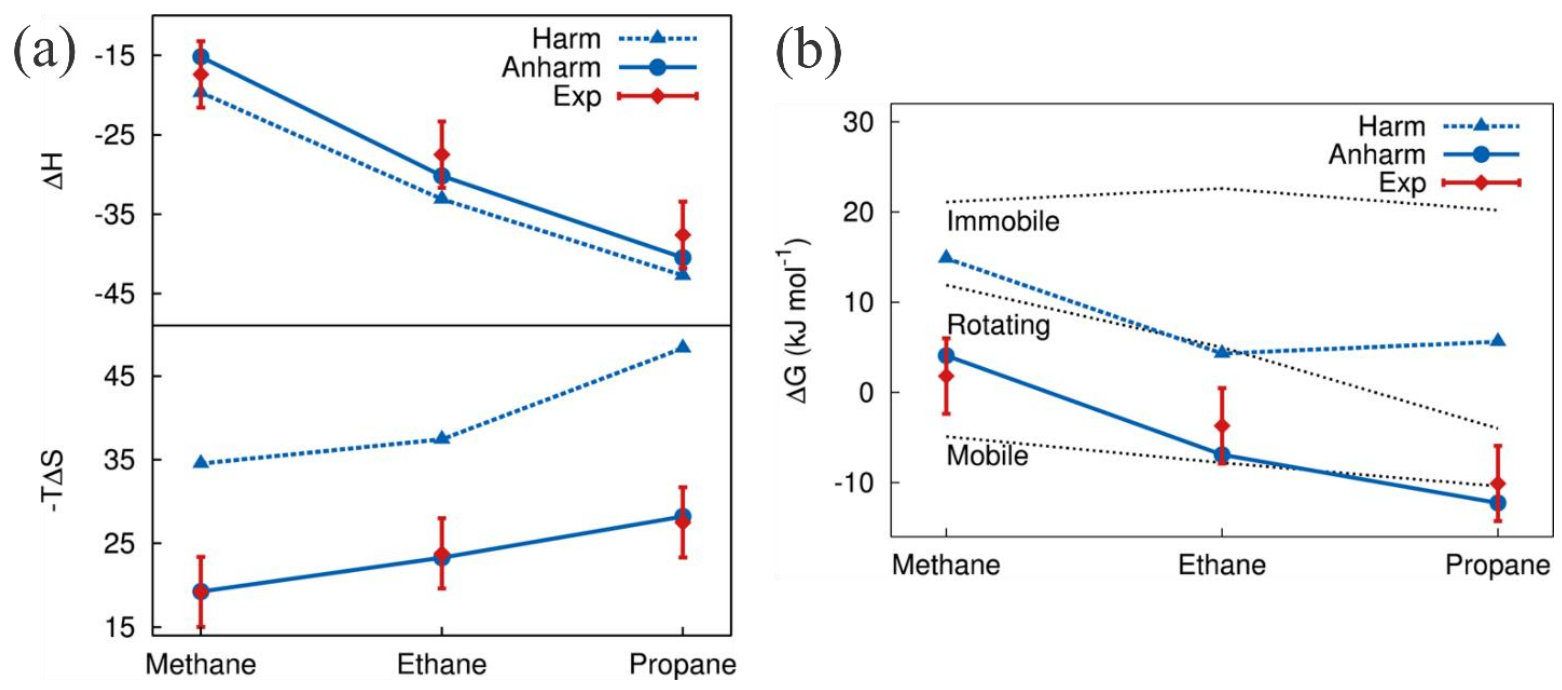

Figure 7. (a) Enthalpy $(\Delta \mathrm{H})$ and entropic term $(-\mathrm{T} \Delta \mathrm{S})$ for the adsorption of methane, ethane and propane in $\mathrm{H}-\mathrm{CHA}$ at experimental conditions $(303 \mathrm{~K}$ for methane, $313 \mathrm{~K}$ for ethane and propane, 0.1 MPa, $\theta=0.5$ ) calculated using harmonic internal normal mode distortions (Harm), anharmonic internal coordinates distortions (Anharm) and experimental data (Exp) (b) Gibbs free energy $(\Delta \mathrm{G})$ for the adsorption of methane, ethane, and propane in $\mathrm{H}-\mathrm{CHA}$ at experimental conditions ( $303 \mathrm{~K}$ for methane, $313 \mathrm{~K}$ for ethane and propane, $0.1 \mathrm{MPa}, \theta=0.5)$ calculated using harmonic internal 
normal mode distortions (Harm), anharmonic internal coordinates distortions (Anharm), experimental data, and simplified adsorption models (black dashed lines for immobile, rotating, and mobile models, respectively). All in kJ/mol. Taken from ref. [80]

The computational cost for performing the anharmonic corrections is about 10 times larger compared to a standard full Hessian computational analysis. Thus when a standard NMA analysis is feasible these additional routines which give a substantial impact on the thermodynamic functions should also be feasible.

Most interestingly, the method of Piccini and Sauer was recently also applied on the methylation reactions already discussed earlier in this perspective.[82] In this seminal contribution they show that reaction rates can be obtained with chemical accuracy in a very systematic way by using a well-designed multistage "divide and conquer" approach, where the level of complexity of the calculations is systematically increased to obtain both chemical accurate enthalpy and entropy contributions to the reaction rates. Figure 8 shows the results for the pre-exponential factors and the rate constants as a function of the alkene length at $623 \mathrm{~K}$. The results were also compared with the earlier results of Svelle et al. and our earlier theoretical results. $[48,56]$ The grey zone in Figure 8 corresponds to a deviation of one order of magnitude compared to the observed values. The agreement between the anharmonic hybrid model proposed by Piccini and experimental values is striking. Furthermore, the corrections are very systematic along the series of alkenes. It is also observed that some of the earlier theoretical models gave also a quite good agreement with experiment although the trend observed in terms of the alkene chain length is not as systematic compared to the more rigorous anharmonic-hybrid model proposed here. The results of Svelle and Van Speybroeck et al. were obtained using a partial Hessian (PHVA) approach where only a limited part of the system was taken into account for the evaluation of the harmonic frequencies. 
The errors introduced by using a harmonic oscillator model on the full system - thus including a full hessian calculation - are much larger. These results show that the surprisingly good agreement between experimental and theoretical data was partly ascribed to a fortuitous cancellation of errors.

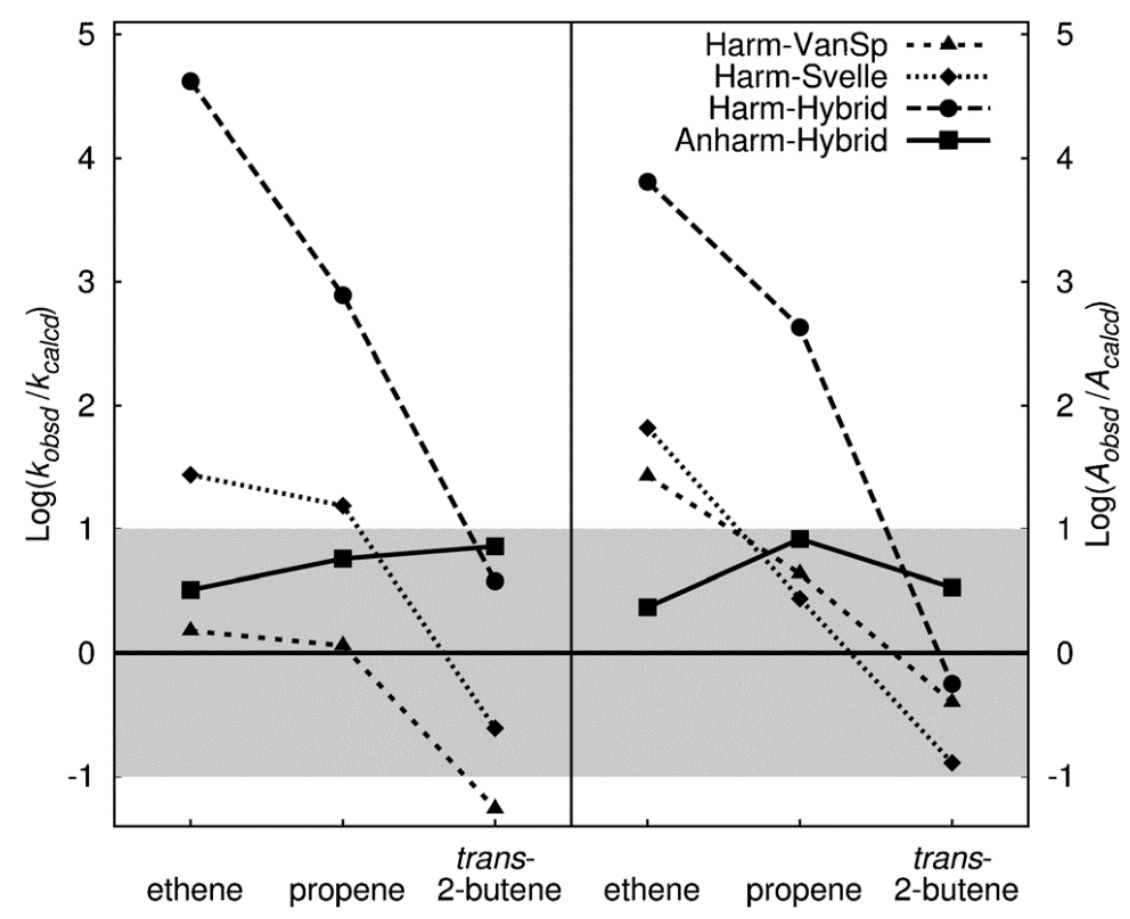

Figure 8. Logarithm of the observed/calculated ratios for the rate constants (left) and preexponentials (right). "Anharm-Hybrid" and "Harm-Hybrid" refer to results calculated with anharmonic and harmonic frequencies, respectively, whereas "Harm-Svelle" refers to harmonic frequencies obtained for a part of the system.[48] The "Harm-VanSp" results [56] were obtained with a 46 tetrahedra cluster model. The gray region represents a deviation of \pm 1 order of magnitude from the observed values. Taken from ref. [82].

It must, however, be noted that the multistep procedure proposed by Piccini is far from trivial to apply on a routine basis, yet the results are seminal as they show that for specific reactions occurring on well-defined active sites, chemical accuracy for reaction rates can be achieved. [47] 
Furthermore, for some specific zeolite topologies and conditions where a larger degree of complexity is introduced, the method as presented here cannot straightforwardly be applied (vide infra).

Interesting modeling advances have been proposed independently by other groups. Noteworthy is the recent approach proposed by Head-Gordon and co-workers, that proposed an alternative method with the ultimate goal to reach the limit of accuracy for low vibrational modes including internal rotations within the assumption that all modes are treated independently. We do not discuss this method extensively here, as it has not been tested extensively on heterogeneously catalyzed reactions.[83]

Finally, it should be mentioned that most approaches to go beyond the $\mathrm{HO}$ approximation focus on the inclusion of anharmonicities and only account for supralinear correlations between different eigenmodes to a lesser extent.[7, 84]

\section{Beyond the HO approach using input from molecular dynamics simulations}

An alternative method to include anharmonicity in the partition functions and derived thermodynamic functions encompasses the use of molecular dynamics (MD) simulations. In MD, the dynamics of the system are followed through discrete integration of Newtonian's equations of motion. The time step must be chosen sufficiently small to accurately sample the fastest motions 
of the system. It is straightforward to proof that the total entropy is the sum of a configurational contribution, i.e. due to the configurational coordinates, and a kinetic contribution, i.e. due to the momenta. In many cases, only the configurational contribution to the entropy is computed as the kinetic contribution is only dependent on the masses of the atoms in the system, which is identical when comparing two configurations of the same system. Several methodologies exist to extract information from MD simulations and estimate entropy.

A first set of methods to quantify entropy uses the so-called Boltzmann-Gibbs-Planck (BGP) expression for (configurational) entropy:

$S=-k \int p(\boldsymbol{q}) \ln p(\boldsymbol{q}) d \boldsymbol{q}$

where $\mathbf{q}$ is the vector of $\mathrm{N}$ coordinates describing the position of all atoms and $p(\boldsymbol{q})$ is the probability density for finding the system in a state with coordinates $\boldsymbol{q}$. Directly evaluating this integral on a grid is practically impossible for systems with many degrees of freedom, as this would require an enormous number of evaluations of the potential energy to construct $p(\boldsymbol{q})$ in the entire phase space. Only for a limited number of theoretical systems for which the probability density is known analytically, or for very small systems, can the expression (6) - usually for didactic purposes - be straightforwardly applied. To reduce such sampling issues, many researchers have opted to distinguish two subgroups of the set of coordinates $\boldsymbol{q}$.[85-87] The first subgroup contains all coordinates that impose relevant differences between various configurations of interest, the second subgroup contains the remainder of the coordinates. Assuming the two sets of coordinates are uncorrelated, i.e. the total probability density can be written as a product of the probability densities of the separate sets of coordinates, the entropy can be written as a sum of two contributions and only the contribution corresponding to the important coordinates are kept for 
further comparison. However, even after reducing the number of degrees of freedom, the complexity of the phase space usually remains too high for direct evaluation of the entropy integral. To truly overcome such sampling issues, one can apply an analytical expression to model the probability density and use the output of regular MD simulations to estimate any unknown parameters or contributions in the expression.

A first model for the probability density consists of approximating the fully correlated N-particle probability density as a density containing only n-particle correlations by means of the Generalized Kirkwood Superposition Approximation.[88] For example, the second-order approximation for the probability density, i.e. containing up to 2-particle correlations, for a N-particle system has the form:

$p^{(2)}\left(x_{1}, \ldots, x_{N}\right)=\frac{\prod_{i<j} p_{2}\left(x_{i}, x_{j}\right)}{\prod_{i} p_{1}\left(x_{i}\right)}$

, in which a superscript denotes the order of the approximation and a subscript $n$ denotes the nparticle correlation contribution. This results in the following approximation for the entropy:

$S^{(2)}\left(x_{1}, \ldots, x_{N}\right)=\sum_{i<j} S_{2}\left(x_{i}, x_{j}\right)-\sum_{i} S_{1}\left(x_{i}\right)$

The entropy contribution $S_{i}$ is computed from the probability density $p_{i}$ using the BoltzmannGibbs-Planck integral (Eq. 6). Including all n-particle correlations results in the mutual information expansion of the entropy proposed by Matsuda et al.[89]

A second model for the probability density, which is often applied, is the so-called quasi-harmonic oscillator approximation $(\mathrm{QHO})$ that accounts for anharmonicity by sampling the exact potential energy surface during a molecular dynamics simulation. As is the case in a normal mode analysis, the probability density is represented by a multivariate Gaussian profile. However, rather than 
using a second-order expansion of the potential energy to model the probability density, one uses the covariance matrix at finite temperature by running MD simulations. The idea is based on the property that the probability density with a given covariance and maximal entropy has a Gaussian profile. As such, it always provides an upper limit to the configurational entropy. This method was first proposed by Karplus and Kushick to estimate the configurational entropy of macromolecules.[86] Later the method was further adapted by Schlitter by adding one crucial quantum mechanically based correction to avoid the divergence for low frequencies in the classical regime as well as deal with the singularity of the covariance matrix[90].

An alternative approach to compute the entropy has recently been used to study ethanol adsorption in ZSM-5 [91] and does not use the Boltzmann-Gibbs-Planck integral. Although the method is also denoted as QHO,[92] it uses a different approach as the one proposed by Schlitter. It can be seen as an extension of the Debye model and interpreted as a continuous-spectrum alternative for QHO. In the proposed method the vibrational density of states (VDOS) using the Fourier transform of the velocity-velocity time-correlation function is computed:

$D(\omega)=\int_{0}^{\infty} e^{-i \omega t}\langle v(\tau) \cdot v(\tau+t)\rangle d t$

The VDOS not only describes spectroscopic properties of the system, but also includes a classical description of the anharmonicity of the system which can be used in harmonic quantum partition functions for vibrations. The vibrational entropy is computed as an integral over the frequencydependent entropy of a harmonic oscillator, weighted by the VDOS:

$S_{v i b}=R N_{D O F} \int_{0}^{\omega_{\max }}\left[\frac{\hbar \omega}{2 k_{B} T} \operatorname{coth}\left(\frac{\hbar \omega}{2 k_{B} T}\right)-\ln \left(2 \sinh \left(\frac{\hbar \omega}{2 k_{B} T}\right)\right)\right] D(\omega) d \omega$

where $N_{D O F}$ represent the number of vibrational degrees of freedom. 
In the study of ethanol adsorption in H-ZSM-5, the free energy of adsorption was calculated from experimentally measured adsorption isotherms and compared to the theoretical predictions. Figure 9 shows some of the most stable structures for the adsorption complexes obtained at $0 \mathrm{~K}$.

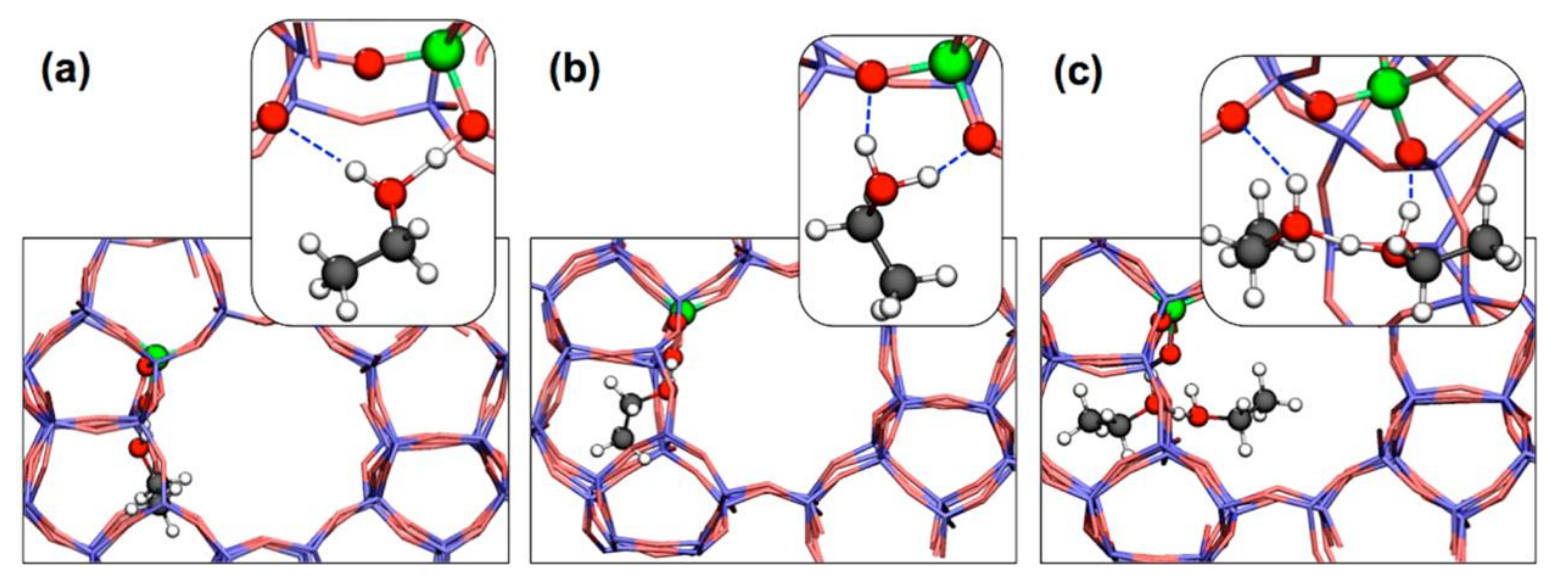

Figure 9. Most stable structures (at $0 \mathrm{~K}$ ) of the adsorbed ethanol monomer of (a) a Zundel-like configuration, (b) a higher energy Eigen-like configuration, and (c) dimer on the Brønsted acid site of H-ZSM-5. Color code: Si (blue), Al (green), O (red), C (gray), H (white), H bonds (dashed blue). Taken from ref. [91].

The authors determined standard adsorption enthalpies, entropies and free energies for the adsorption of one and two ethanol molecules by applying different theoretical methods as well as from experimental measurements. Overall the harmonic oscillator model seemed to overestimate the free energies of adsorption when comparing them to the experimental data, whereas the QHO model gave values which are much closer to the experimental data. It should however be noted that the corrections for the entropy and enthalpy are not as systematic as expected and contradict earlier adsorption studies that compare beyond-HO methods with the HO approach, $[63,80]$ in which it is systematically stated that the HO approach systematically overestimates entropy losses and therefore predicts less stable adsorption of guest molecules in zeolites. The calculated data 
were in good agreement with measured infrared spectra and calorimetric data. A reduction of the error on calculated adsorption free energies from $20-50 \mathrm{~kJ} / \mathrm{mol}(\mathrm{HO})$ to $\pm 10 \mathrm{~kJ} / \mathrm{mol}$ was achieved. More benchmarking using this method and comparison with other methods to account for anharmonic corrections would be very interesting in the future.

The QHO approach to compute entropy is being applied in a variety of fields, for example for the prediction of the stability and thermodynamic properties of mixtures such as $\mathrm{CO}_{2} / \mathrm{H}_{2} \mathrm{O}$, [93] and $\mathrm{HCl}\left(\mathrm{H}_{2} \mathrm{O}\right)_{6}$ clusters, [94] and in the field of computational drug design and discovery.[7]

Finally, a last group of methods does not directly compute the entropy, but instead first computes the free energy and the internal energy, from which the entropy is easily derived. The internal energy is easily extracted from molecular dynamics or Monte Carlo simulations as it represents a simple average of the Hamiltonian (or the potential energy in case only the configurational energy is of interest). Computation of the free energy on the other hand, faces the same sampling issues as the entropy. To compute the free energy, one usually applies the (quasi)harmonic approximation or advanced sampling methods (see section 5), but in some cases other approaches are used. For example, Head and co-workers identified the dihedral angles of a protein molecule as most relevant coordinates, while all other were considered unimportant.[95] The torsional partition function is then written as a sum of contributions from a priori identified low-energy conformations. Finally, the contribution for each conformation is estimated by means of a Monte Carlo scheme.

One point that is often overlooked in classical molecular dynamics runs, is the importance of nuclear quantum effects.[96, 97] For reactions involving light atoms such as hydrogen, these effects will grow in importance. Recently Ceriotti and co-workers developed a Python interface for using path integral MD simulations in combination with electronic structure programs.[98] 
Another methods consists of generating quasi-classical trajectories (QCT) starting from a transition state with the initial velocities of all atoms based on quantum mechanical vibrational populations, including zero point energy (ZPE). This is particularly important for systems where ZPEs are large or where $\mathrm{C}-\mathrm{H}$ bonds are vital to the reactivity. The QCT method has proven its power in a variety of applications.[23, 99-102]

\section{Advanced molecular dynamics simulations to estimate entropy for zeolite catalyzed reactions.}

As stated before, MD-based techniques allow to go beyond the harmonic oscillator approximation. However, the determination of free energies and entropies of chemical reactions from advanced molecular dynamics simulations is computationally very challenging. First as we want to simulate chemical reactions where bonds are broken and formed, one needs to resort to molecular dynamics simulations on potential energy surfaces that have been determined by first principle or quantum mechanical based methods. In practice DFT methods are the method of choice to treat such large systems, as they offer a favourable balance between accuracy and computational efficiency. Compared to force field based molecular dynamics routines, these methods are however extremely computationally expensive. This is especially true when considering the fact that extensive sampling is necessary to get converged statistical quantities. Overall the dynamics of the system spans a wide range of characteristic time scales. Chemical transformations are rare events as their probability of occurring is very low, although once it occurs the event may be followed during a regular MD run of a few picoseconds. Various advanced sampling techniques have been introduced to obtain the free energy of the system along some well-defined regions of the free 
energy surface.[103-112] However, in reality the free energy surface for a zeolite catalyzed reaction is very complex as schematically illustrated in Figure 10. The hypothetical free energy map shown in Figure 10 is comparable to a topographical map used when hiking the mountains. The challenge consists in localizing these passages representative for making the transition from one free energy basin to another.

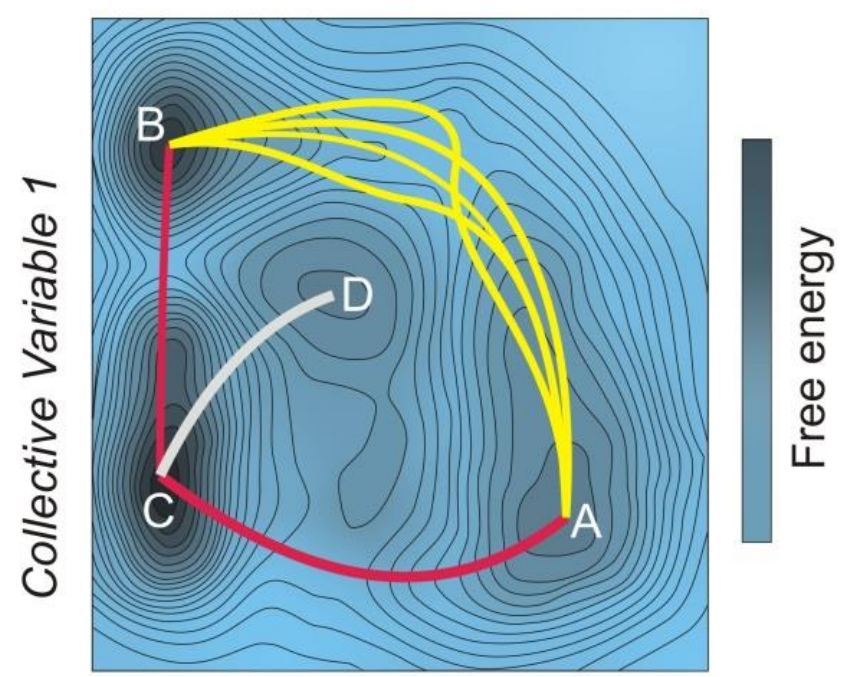

Collective Variable 2

Figure 10. Hypothetical two dimensional free energy surface as a function of two collective variables. Minima A and B are connected by direct and two-step reaction pathways. At operating conditions, multiple pathways might be followed along the same free energy valley as illustrated by the yellow paths for the direct conversion of A to B. Intermediate C can also lead to byproduct D. Adapted from ref. [13].

At operating conditions for zeolite-catalyzed reactions, one typically has to account for the presence of additional guest molecules, flexibility of the zeolitic framework, configurational degrees of freedom of guest molecules in the zeolitic pores and the existence of competing 
pathways. Altogether, the aforementioned factors contribute to the complexity of the free energy landscape of the system at high temperature. To capture this complexity, advanced molecular dynamics simulations are now gradually making their entrance within the field of zeolite catalysis. Hereafter we discuss some noteworthy examples which clearly show the power of advanced molecular dynamics simulations to probe the reactions of interest at true operating conditions.

As has been mentioned before, static methods are not appropriate to give a proper qualitative insight into the observed nature of intermediates at operating conditions in some cases. This was very recently shown for adsorption intermediates observed during alkene cracking, a process that typically takes place at very high temperatures around $500{ }^{\circ} \mathrm{C}$. Retrieving insight into the exact nature of the intermediates is also very challenging from experimental point of view as some species are short-lived and may - even at low temperatures - easily undergo transformations, making it very hard to follow alkene cracking reactions even with state-of-the-art experimental techniques. However, it should be noted that in the last decades major progress has been made in the field of in-situ and operando spectroscopy which now allows tracking important reaction paths and species of complex catalytic transformations. We refer the interesting reader to some dedicated reviews on the topic.[113-118] When an alkene interacts with the Brønsted acidic site, it forms a $\pi$-complex, which may be protonated yielding a chemisorbed species (Figure 11). The nature of the chemisorbed species has been heavily debated, as it might occur as a covalently bound alkoxide species or as a carbenium ion.[119] 


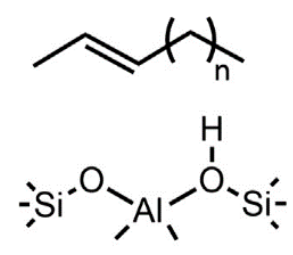

1

$1 \underset{\text { physisorption }}{\stackrel{\text { chemisorption }}{\rightleftharpoons}}$

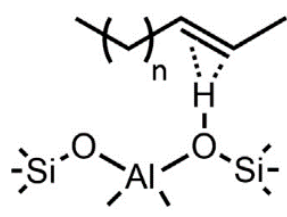

2
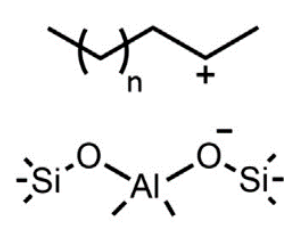

3
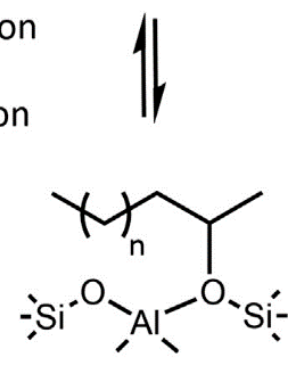

4

Figure 11. Various complexes that can be formed by an alkene in the presence of a Brønsted acid site. 1: Van der Waals complex, 2: $\pi$-complex, 3: carbenium ion, 4: alkoxide. Taken from ref.[120].

Most theoretical studies so far were based on static simulations and geometry optimizations at the $0 \mathrm{~K}$ surface. Entropy contributions were added using the routines relying on the harmonic oscillator approach as described earlier in this perspective. A particularly interesting study on the possible existence of carbenium ions was performed by Tuma and Sauer.[50, 121, 122] They evaluated the stability of the tert-butyl cation and alkoxide species in H-FER and included also for the first time entropy effects. Important temperature effects were found on the relative stability of the alkoxide versus the carbenium ion. Electronically, the tert-butoxide was more stable than the tert-butyl cation, but for temperatures higher than $120 \mathrm{~K}$ the carbenium ion becomes more stable. The results from this study show that the persistence of intermediates within zeolites depends largely on the conditions and cannot be evaluated solely on basis of electronic energies.

Very recently we performed extensive static and molecular dynamics simulations on the adsorption of both linear and branched pentene in H-ZSM-5 at catalytic cracking conditions.[120, 
123] The results are surprising and show a clear influence of entropy on the stability of the prevalent intermediates. An extensive number of regular MD simulations were performed using DFT to sample the potential energy surfaces at three different temperatures $\left(50,300\right.$ and $\left.500{ }^{\circ} \mathrm{C}\right)$. We systematically started from different starting adsorption complexes and evaluated the behavior of the observed species during the entire MD simulations. The results are schematically depicted in Figure 12.

When starting from a linear alkoxide (Figure 12a), this species remains stable during the course of the simulation at low temperatures. However, when temperature is increased, entropy effects favour the more loosely bound physisorbed structures namely the $\pi$-complex or Van der Waals complex (see Figure 11). Apparently the carbenium ion is hardly observed for linear alkenes. To further verify this observation, we started a new series of MD simulations from the carbenium ion (Figure 12b). This complex almost immediately rearranged to a physisorbed complex. Thus for linear alkenes carbenium ions are unlikely to occur even at cracking temperatures. The relative stability of the alkoxide versus the $\pi$-complex depends, however, quite heavily on the applied simulation method. Using the static method, the relative stability of alkoxides versus $\pi$-complexes is also not correctly assessed. Static methods tend to overestimate the stability of $\pi$-complexes. In molecular dynamics simulations the average distance of the double bond of the $\pi$-complex to the Brønsted acid proton is larger than in static simulations yielding an overall weaker adsorption. 
(a)

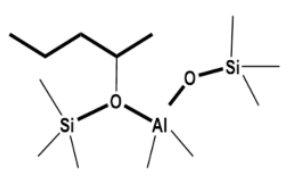

$50^{\circ} \mathrm{C}$

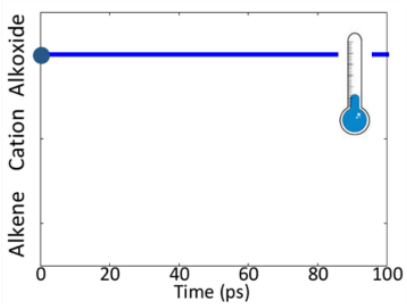

(b)

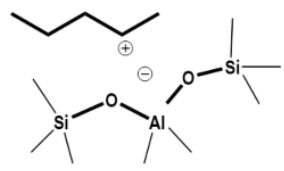

$50^{\circ} \mathrm{C}$

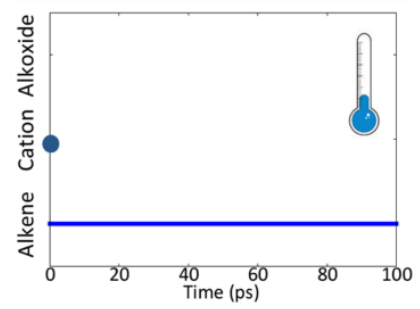

(c)

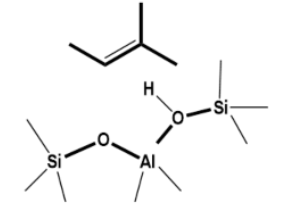

Branchedpentene
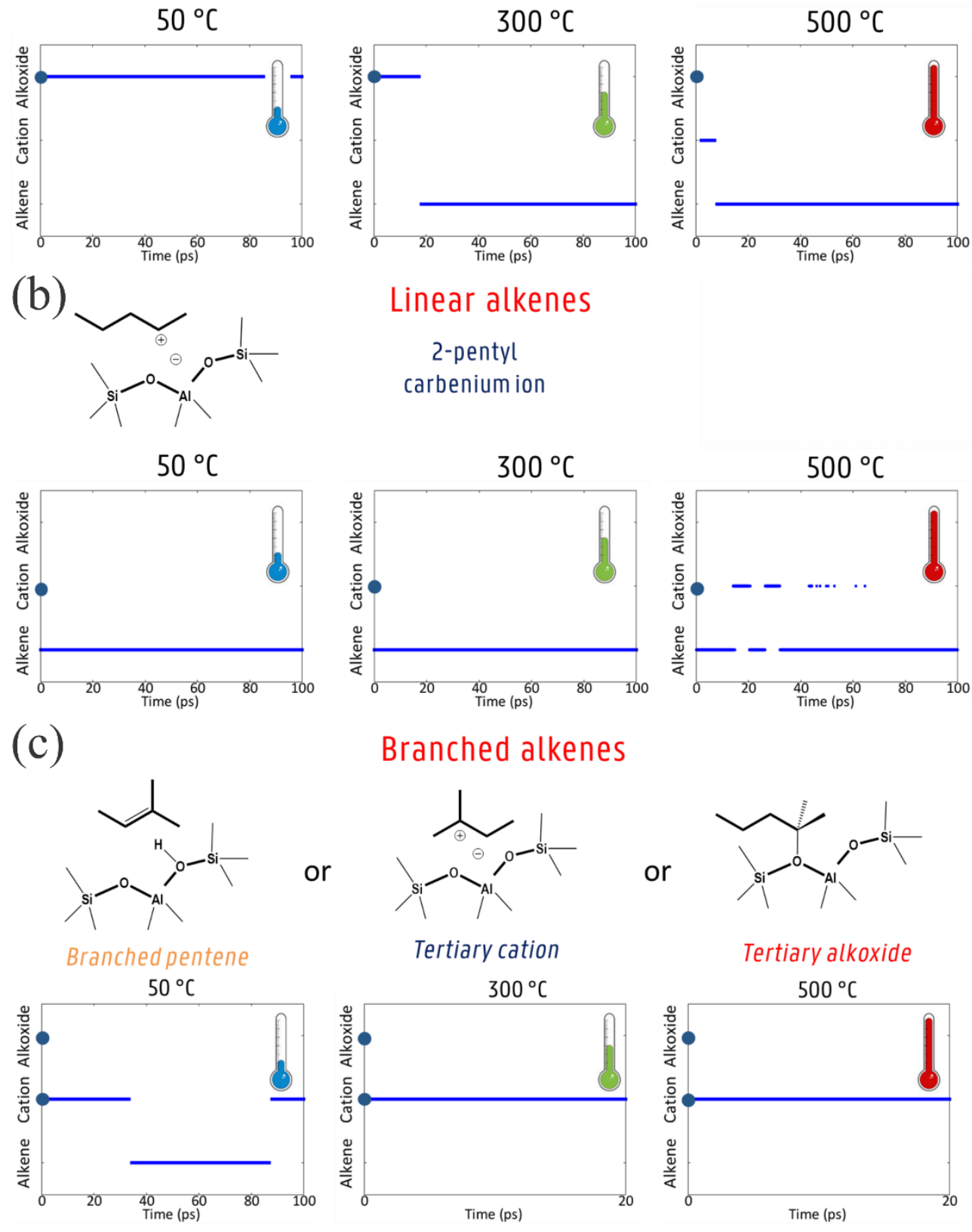

Linear alkenes

2-pentyl

carbenium ion
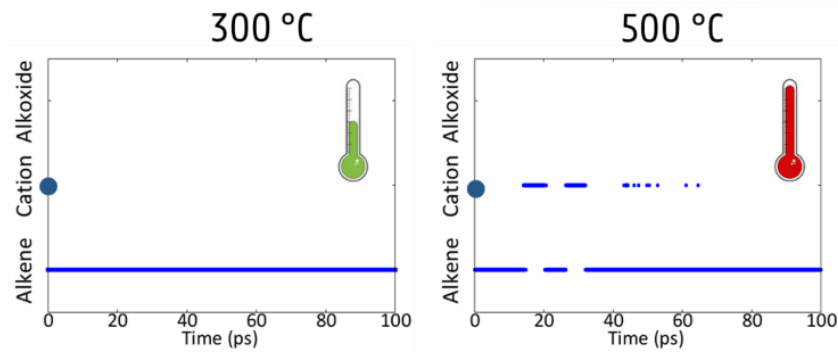

Branched alkenes

or

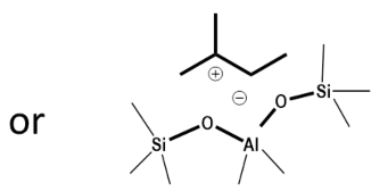

Tertiary cation

$300^{\circ} \mathrm{C}$

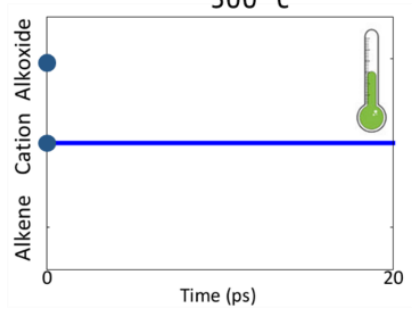

or

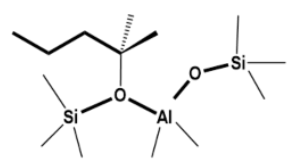

Tertiary alkoxide

$500^{\circ} \mathrm{C}$

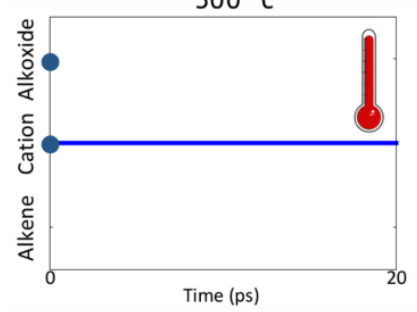

Figure 12. Time-evolution of different linear $(a, b)$ and branched (c) pentene intermediates during MD simulations at 50, 300 and $500{ }^{\circ} \mathrm{C}$. 
For branched alkenes, similar MD simulations were performed which gave unprecedented insights into the nature of the adsorbed species at cracking temperatures.[120] When starting from the alkoxide (Figure 12c), this species quickly rearranges to the carbenium ion. At low temperatures, the physisorbed complex is also sampled for an appreciable amount of time, however at higher cracking temperatures the carbenium ion becomes the prevalent species. Our conclusions have far reaching consequences for evaluating reaction mechanisms at these high temperatures. One should start from the carbenium ions rather than from alkoxides. Entropy plays a decisive role in the stability of these carbenium ions and in general more loosely bound complexes. As no explicit bond is formed between the adsorbate and the zeolite lattice, these species are entropically favored at higher temperatures.

As mentioned previously, estimating the entropy directly from the Boltzmann-Gibbs-Planck integral (Eq. 6) is quite challenging as it requires evaluations of the energy on a grid representing the entire phase space, which imposes an immense computational cost for all but the smallest systems. Instead, molecular dynamics and Monte Carlo simulations aim to efficiently sample those regions of phase space that have non-zero contributions to the partition function and hence the free energy and entropy. However, even within these simulation methodologies, severe sampling issues remain, especially when two relevant states (i.e. basins in the free energy profile) are separated by a high free-energy barrier. Since such barriers cannot easily be overcome in the usual temperature range - i.e. room temperature - MD or MC simulations will usually remain in the initial state without sampling the other relevant states. To remedy such sampling issues, one can use molecular simulations with enhanced sampling techniques (eg. metadynamics, umbrella sampling, thermodynamic integration, ...), which aim to enhance the sampling of states separated by high free energy barriers. Such techniques usually start by defining a collective variable (CV), i.e. a 
degree of freedom that connects the two stable states and defines the reaction path. Then the procedure either imposes a bias potential along the $\mathrm{CV}$ that lowers the barrier (eg. metadynamics,[107] umbrella sampling,[124] ...), or by constraining the value of the CV and performing a scan along the reaction path (eg. thermodynamic integration,[125] free energy perturbation,[126] ...). The major drawback of such methods is, however, that it requires an adequate definition of the collective variable, which is all but a trivial task and the results usually depend on the choice of the CV.[127]

Previous case study is a clear example of an application where static methods are too restricted to obtain insight into the process at operating conditions. To further illustrate the strength of an advanced molecular dynamics approach, we highlight two case studies describing the methylation of aromatic and aliphatic molecules in a zeolitic environment.

The first case study concerns the completion between the concerted and stepwise pathways for benzene and propene methylation. As mentioned earlier in this perspective, methylation reactions may occur either in a concerted or stepwise fashion, i.e. methanol directly transfers its methyl group to a hydrocarbon or the reaction goes through a framework-bound methoxide intermediate. The selectivity for one or the other mechanism has earlier been suggested to be entropy driven.[10, 11] The reactions were studied in the unidirectional AFI-structured H-SSZ-24, H-SAPO-5 and TON-structured H-ZSM-22 materials. A DFT-based dynamical approach was applied which enables mimicking reaction conditions as close as possible and studying the competition between two methylation mechanisms in an integrated fashion. Referring back to Figure 10, this means that we explicitly studied the competition between paths that directly connect free energy basins A and $\mathrm{B}$ and paths that connect $\mathrm{A}$ and $\mathrm{B}$ via intermediate $\mathrm{C}$. This competition was simulated by a large number of metadynamics simulations to obtain free energy barriers for every reaction step. As can 
be seen from Table 2 , we found that only at higher temperatures, i.e. $350{ }^{\circ} \mathrm{C}$, the stepwise pathway might become competitive with the concerted methylation, which indeed confirms the distinct entropic effects for both mechanisms.[12] Indeed, the intermediate release of a water molecule during methoxide formation seems to be beneficial at high temperatures. It can also be observed that the competition between the two pathways is largely influenced by the specific zeolitic material and hydrocarbon species. We observed in particular that for benzene methylation at 350 ${ }^{\circ} \mathrm{C}$ in H-SAPO-5 and H-ZSM-22 that a concerted methylation step and methoxide formation are nearly equally high activated, whereas the concerted mechanism always prevails in H-SSZ-24.

Table 2. Free energy barriers and reaction energies in $\mathrm{kJ} / \mathrm{mol}$ for the concerted $\left(\Delta \mathrm{G}^{\ddagger}\right.$ concerted $)$ and first step of the stepwise methylation $\left(\Delta \mathrm{G}^{\sharp}{ }_{\text {step } 1}, \Delta \mathrm{G}_{\mathrm{r}, \mathrm{step} 1}\right)$ of benzene by 1 methanol molecule at $250{ }^{\circ} \mathrm{C}$ and $350{ }^{\circ} \mathrm{C}$ in H-SSZ-24, H-SAPO-5 and H-ZSM-22.

\begin{tabular}{lc|c|cc}
\hline & & Concerted & \multicolumn{2}{|c}{ Stepwise } \\
& & $\Delta \mathbf{G}_{\text {concerted }}^{\ddagger}$ & $\Delta \mathbf{G}_{\text {step1 }}^{\ddagger}$ & $\Delta \mathbf{G}_{\mathbf{r}, \text { step1 }}$ \\
& & $(\mathbf{k J} / \mathbf{m o l})$ & $(\mathbf{k J} / \mathbf{m o l})$ & $(\mathbf{k J} / \mathbf{m o l})$ \\
\hline H-SSZ-24 & $350{ }^{\circ} \mathrm{C}$ & $134 \pm 3$ & $160 \pm 3$ & $46 \pm 4$ \\
& $250{ }^{\circ} \mathrm{C}$ & $136 \pm 1$ & $179 \pm 3$ & $48 \pm 4$ \\
\hline $\mathbf{H}-S A P O-5$ & $350{ }^{\circ} \mathrm{C}$ & $156 \pm 1$ & $162 \pm 3$ & $3 \pm 1$ \\
& $250{ }^{\circ} \mathrm{C}$ & $165 \pm 4$ & $180 \pm 5$ & $18 \pm 5$ \\
\hline $\mathbf{H}-Z S M M-22$ & $350{ }^{\circ} \mathrm{C}$ & $151 \pm 2$ & $148 \pm 3$ & $31 \pm 2$ \\
\hline
\end{tabular}

Further, we also addressed the impact of an additional methanol molecule on the kinetics of benzene methylation. The presence of such additional protic guest molecule mainly influences the 
ease of methanol protonation prior to reaction. We observed two types of reaction paths: paths in which the additional methanol actively participates and paths in which it doesn't. It seemed that the influence of such assistance on the activation free energy is not straightforward. Indeed, we earlier found that additional methanol molecules might assist methoxide formation from methanol in H-ZSM-5 whereas no effect was found on the same reaction from dimethyl ether (DME).[128] In contract, the presence of multiple methanol molecules in the surroundings of the zeolite's active site were found to slow down benzene methylation in H-ZSM-5.[22] Moreover, it was also found that methanol assists methoxide formation reactions more efficiently than water molecules.[14] It became clear that additional molecules have a very complex behaviour at operating conditions and a delicate balance between a multitude of effects determines whether or not they have a beneficial effect on the activation free energy. Typically, multiple protic molecules form protonated clusters near the Brønsted acid site as depicted in Figure 13, which exhibit different reactivity compared to single molecules.[22, 128] The formation of such clusters is of course accompanied by a significant entropic penalty which determines the optimal size of each cluster.[22] Further dedicated studies on the impact of guest molecule loading and in particular the feed composition on the reaction mechanism are recommended from both an experimental and theoretical viewpoint. 


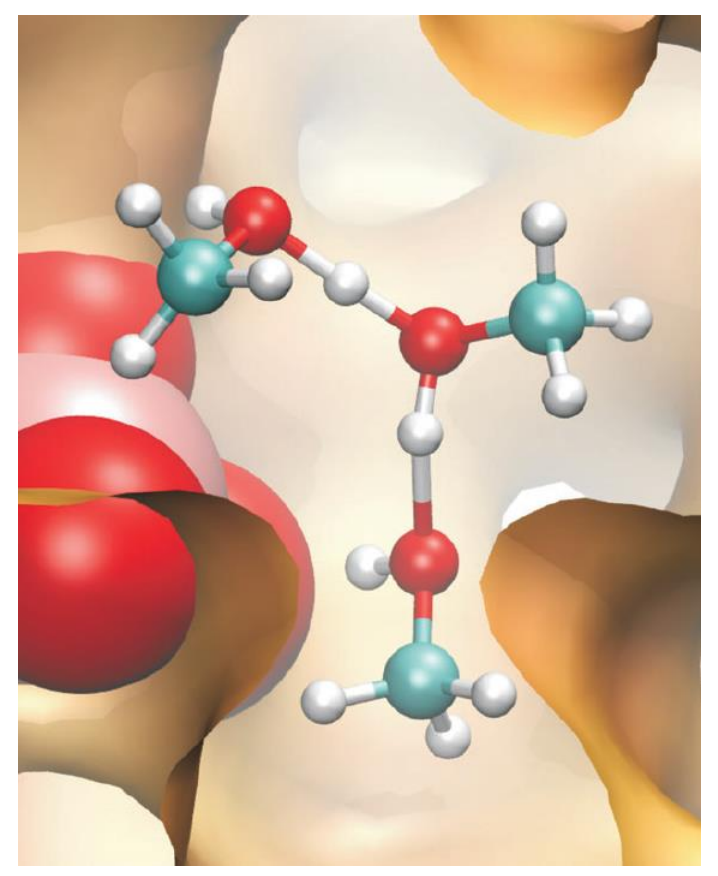

Figure 13. Protonated methanol trimer inside the channel intersection of H-ZSM-5. The AlO4 site is represented as van der Waals spheres, with the central Al atom colored pink. Taken from ref. [16]

An alternative approach to assess the influence of operating conditions on the competition between the concerted or stepwise methylation of alkenes in H-ZSM-22 was proposed by Brogaard et al.[11] They addressed the debate by combining TAP kinetic experiments, static DFT calculations and micro-kinetic modeling. The micro-kinetic modeling of the alkene methylation reactions was done using CatMAP, to obtain a steady-state solution of a set of coupled rate equations corresponding to a series of elementary reaction steps.[129] As such, the product formation rate for each individual mechanism can be obtained as function of the operating conditions, i.e. temperature and pressure, as shown in Figure 14. It could be concluded that at temperatures below $350 \mathrm{~K}$, the concerted methylation pathway prevails over the stepwise whereas the preference reverses with increasing temperature is increased. Moreover, the pathway transition temperature 
increases with pressure. Hence, at conditions of typical MTH experiments (temperatures above $600 \mathrm{~K}$ and total pressures below $1 \mathrm{bar}$ ) the stepwise pathway seems to be 3 to 4 orders of magnitude faster than the concerted pathway. At typical industrial conditions with total pressures around 20 bar,[130] the micro-kinetic model predicts a closer competition between the pathways. The authors identified the entropic effect of the intermediate release of a water molecule as the origin of this shift in dominant methylation mechanism. This study once again illustrates the complex task to disentangle the reaction mechanism and directly compare experimentally measured kinetic parameters with theoretical data (see section 2 of this perspective).
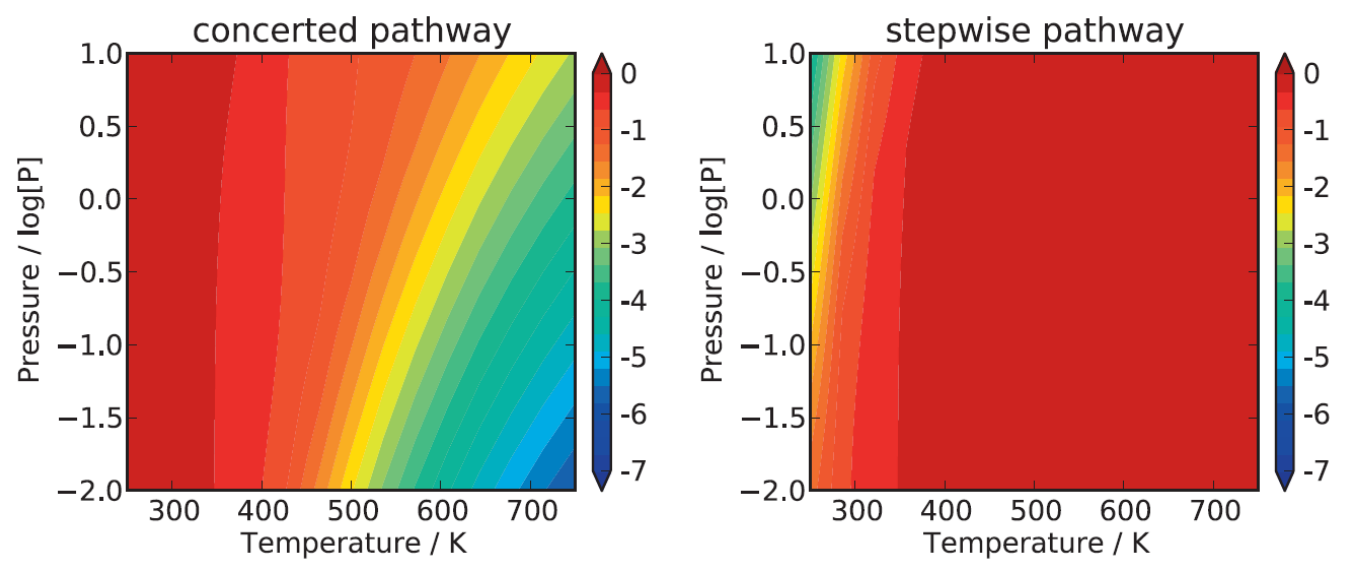

Figure 14. The rate of product formation (logarithmic scale) along each of the concerted and stepwise pathways relative to the total rate of propene production from methanol/DME and ethene in H-ZSM-22. Taken from ref. [11]

In the second case study, the methylation of benzene in the presence of two methanol molecules at $350{ }^{\circ} \mathrm{C}$ in the large-pore $\mathrm{H}-\mathrm{SSZ}-24$ material was investigated by metadynamics simulations.[13] Because the framework is prone to deprotonation prior to reaction, any further reaction can start from physisorbed methanol, a single protonated methanol molecule, or a protonated methanol dimer (vide supra). Moreover, the H-SSZ-24 material exhibits large pores, giving a large 
configurational freedom to the relatively small adsorbed molecules, i.e. methanol and benzene. Because of these two reasons, reactants can adopt many configurations, leading to a relatively flat potential energy surface. For this system, the metadynamics approach captures the complexity of the process adequately by sampling multiple paths in the same free energy valley (cfr. yellow paths in Figure 10). Indeed, for the three sampled reaction steps a multitude of nearly isoenergetic transition states could be observed as displayed in the snapshots shown in Figure 15. This could be attributed to the large available space in the large pore AFI material, resulting in the existence of multiple transition paths connecting the free energy minima. Indeed, in the calculated free energy surfaces broad transition regions were observed. It should be noted that his effect can only be observed with a dynamical approach.

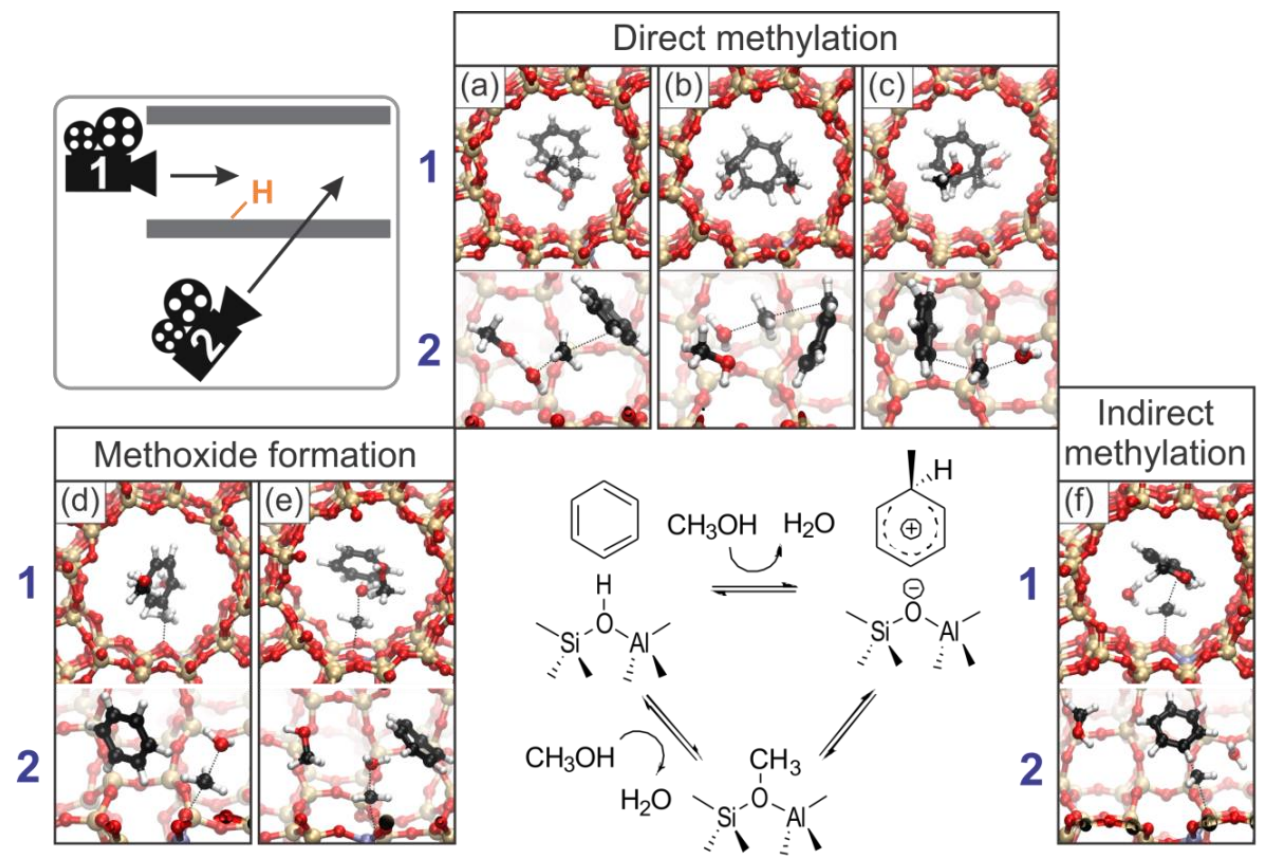

Figure 15. Snapshots of the concerted $(\mathrm{a}, \mathrm{b}, \mathrm{c})$ and stepwise methylation $(\mathrm{d}, \mathrm{e}, \mathrm{f})$ of benzene by 2 methanol molecules in H-SSZ-24 at $623 \mathrm{~K}$, seen in the direction of the 1D channel (camera viewpoint 1) and in a cross section of the channel (camera viewpoint 2). Taken from ref. [13] 
Finally, we mention some other successful examples of the application of advanced MD simulations to capture important entropic effects in zeolite catalysis.

Benco et al. studied propane dehydrogenation over Zn-exchanged mordenite.[17] They made an explicit comparison of the description of entropy loss during propane dehydrogenation by static and thermodynamic integration based on constrained MD simulations and found major differences. The harmonic transition state theory clearly underestimated the entropy loss during the transition from a loosely bound physisorbed propane to a strongly bound $\mathrm{Zn}$-propyl complex because soft degrees of freedom such as hindered rotations and translations are not well described in a harmonic approximation (vide supra).

An explicit entropy estimation based on constrained MD and thermodynamic integration was made by Bučko et al. for monomolecular propane cracking over chabazite.[19] While the free energies of activation and reaction are direct output from the thermodynamic integration procedure, the internal energy difference can be computed as the average energy during the simulation. The difference between the free energy and internal energy contributions then yields the entropic term. It should however be noted that caution is warranted with this approach as error bars on energies during MD simulations can be quite significant, resulting in significant error bars on the internal energy and entropy. Alternatively, one could think of performing free energy calculations at two temperatures to estimate the entropic contribution. This simplistic approach, however, requires the assumption that enthalpic and entropic contributions are independent of the temperature in the considered interval which is not necessarily true. Indeed, for typical hydrocarbon conversions in zeolites enthalpic and entropic contributions to adsorption and activation free energy barriers are not constant.[131] 
Entropy can also be computed from metadynamics simulations during which the energy of the system has been used as one of the collective variables.[132] This allows to determine the entropy at all temperatures from a single simulation, without any approximations. The drawback of this technique is that only very small perturbations under the form of Gaussian hills can be allowed to ensure a physically relevant sampling, which makes this technique very computationally demanding for realistic catalytic systems.

Finally, Bell and co-workers demonstrated that $0 \mathrm{~K}$ potential energy surfaces alone cannot accurately predict product selectivities in cracking of alkanes over H-MFI as selectivity in zeolite catalysis is largely determined by dynamical effects in high-temperature reaction pathways.[23, 99] Therefore they generated quasi-classical trajectories (QCT) starting from a transition state (see section 4).

\section{Conclusions and perspectives}

Within this perspective we have shown the importance of entropy for the accurate description of adsorption and reaction kinetics of zeolite catalyzed reactions. The role of entropy was already acknowledged to be very important within the field of zeolite catalysis and more generally for heterogeneous catalyzed reactions. However, the explicit quantification of entropy both from experiment and theory has proven to be a major challenge. From a modeling perspective most attention was given so far to the accurate determination of reaction mechanisms and the determination of reaction barriers. The estimation of the free energies of the reaction and more particularly the entropy contributions to the various steps of the reaction was lagging behind for a very long time. However, in the last couple of years, major advances have been made in the field. 
First of all Campbell and Sellers, succeeded in quantifying the importance of entropy by reanalyzing a massive number of adsorption isotherms and calorimetric data.[1] This allowed to quantify the entropy of adsorbed molecules on surfaces and to conclude that entropies of adsorption are much larger than generally believed.

From a modeling perspective reaction kinetics has mostly been estimated using Transition State Theory, where only a limited number of points on the potential energy surface are taken into account. Despite its simplicity major advances have been made during the last decade to predict reaction rate constants with chemical accuracy using TST. At first instance attention was mostly given to determining reaction enthalpies of adsorption and activation with chemical accuracy. Seminal papers appeared by the group of Sauer and co-workers.[48-50] They showed that reaction enthalpies of zeolite catalyzed reactions could be determined with chemical accuracy provided well designed multi-step procedures were used which systematically improve the complexity of the calculations. These methods come at a substantial computational cost and cannot be used on a routine basis. Within the concept of transition state theory also entropy contributions can be evaluated by performing a so-called normal mode analysis. In this case all internal motions of the system are described as a superposition of independent harmonic oscillators. For the methylations of alkenes in H-ZSM-5 we showed that accurate reaction rate constants could be achieved using a computationally attractive model. However, care must be taken to generalize these conclusions for any zeolite catalyzed reaction. First of all, the reaction scene at operating conditions is often far more complex than simulated in these studies. In such cases more advanced simulations methods should be used to go beyond a static approach.

Even within such static approach the harmonic oscillator model has clear weaknesses. For low vibrational modes or so-called soft modes, the HO model breaks down in the limit of frequencies 
reaching zero. In these cases, the vibrational entropy diverges. Furthermore, it has proven to be a very intensive and sometimes even impossible task to converge structures to a level where all frequencies are positive. Even when this goal is achieved, the numerical noise associated with the obtained frequencies is large and as these low lying frequencies have a particularly large contribution to the vibrational entropy, the induced errors on the entropy may be unacceptably large.

A series of methods have been proposed to circumvent this problem, ranging from simple numerical solutions without good physical motivation to more ingenious algorithms. Noteworthy are the mobile adsorbate method proposed by some of us, where some of the low vibrational partition functions are replaced by free rotational and/or translational contributions. Using the $\mathrm{HO}$ approach, adsorbates are generally too much immobilized on the surface and thus the entropy losses are overestimated. Using the mobile adsorbate method prevents this artificial loss of entropy by allowing some degrees of rotational and/or translational freedom. The usage of this method to transition state theory might however not be trivial. This is especially true for large pore zeolites with a lot of configurational freedom for the guest molecules.

Another suite of very interesting techniques was proposed recently by Piccini and Sauer. They proposed a set of ingenious theoretical algorithms that solve various problems simultaneously. Initially they proposed a re-optimization step of the various complexes by performing geometry optimizations in normal coordinates rather than in Cartesian coordinates. It was proven that the numerical uncertainty on the obtained frequencies is much smaller in this case. Furthermore, they suggested a theoretical algorithm to include anharmonic corrections in a systematic way. The method comes at a reasonable computational cost and thus has the potential to be applied on a broad set of systems within heterogeneous catalyzed systems. They showed for the adsorption of 
methane, ethane and propane in H-SSZ-13 that systematic corrections are obtained which bring the theoretical prediction closer to the experimental values. For the methylation of alkenes in $\mathrm{H}-$ ZSM-5 they achieved chemical accuracy for the reaction rates in a systematic way using a multistep approach that systemically increases the level of complexity in the theoretical models to achieve both chemical accurate enthalpy and entropy barriers. Yet their method cannot be regarded as a standard procedure, as the computational expense is enormous. However, it opens very interesting perspectives as chemical accuracy for reactions taking place at a well-defined active site have come within reach.

We also showed examples of where one needs to go beyond static methods, as the reaction conditions and reaction mechanisms are far more complex at operating conditions. One particular case that attracted much attention in literature concerns the nature of intermediates observed at cracking conditions in zeolites. These processes typically take place at very high temperatures and it is very difficult to obtain information on the nature of the intermediates at these conditions. Intuitively one expects that more loosely bound species such as free alkenes or even carbenium ions might become more stable at high temperatures since they are entropically favored compared to species covalently bound to the zeolite framework. Using a series of advanced molecular dynamics simulations at a set of different temperatures this was for the first time explicitly proven recently. At lower temperatures alkoxides are prevalent intermediates whereas at higher temperatures and depending on the specific alkene carbenium ions or free alkenes become more probable. These conclusions have far reaching consequences as modeling studies on cracking reactions should start from the correct intermediates at operating conditions. These methods show a lot of potential although further methodological improvements are necessary to extract explicitly entropy contributions from these simulations. 
Advanced molecular dynamics studies have also been applied to study zeolite catalyzed reactions in presence of many guest molecules and taking place in large pore zeolites. These are typically examples where not only one transition state can be localized, but a lot of isoenergetic points exist in the transition region. In the case of more guest species this must be related to many possible configurations of the various molecules in the pores of the confined material. For the large pore zeolite, transition states typically are also subject to a lot of configurational freedom. In such case it becomes essential to sample the phase space extensively and to get track of the configurational entropy associated with the reaction. We applied these methods successfully to obtain new insights into the preferred reaction mechanism as higher temperatures, taking into account the flexibility of the zeolite lattice, the presence of multiple guest molecules, temperature effects and the existence of competing reaction pathways. It should however be noted that these advanced molecular dynamics simulations come at a huge computational cost. Furthermore, serious methodological hurdles have to be overcome to apply these methods in a more standard way on zeolite catalyzed reactions. Various methodological advances are continuously proposed by renowned theoretical groups.[127, 133-138] The true challenge for the next coming years is the implementation and modification of these methods to heterogeneously catalyzed reactions. Every modeling study using advanced molecular dynamics studies so far is confronted with new unexpected difficulties. For example, the definition of the collective variables maybe very troublesome, or convergence of the free energy for many degrees of freedom is also very challenging. As all simulations have to be performed using first principle based methods, another challenge is the computational cost associated with these simulations.

Moreover, recent developments in the field of machine learning have paved the way for efficient total energy calculations. A prerequisite for the application of any machine learning technique is 
the identification of good descriptors for the system under study.[139-145] A next step could be to extend these principles to normal mode analysis and entropy calculation in general to allow the efficient screening of new catalyst materials. For such high-throughput calculations construction of a database and identification of good descriptors is essential.

In any case during the last decade we have seen an enormous progress in modeling with chemical accuracy reaction kinetics and more particularly the entropy contributions to the various reaction steps. More recently various noteworthy developments are being made which also make the accurate modeling of entropy within reach.

\section{Acknowledgements}

We thank the Foundation of Scientific Research - Flanders (FWO), the Research Board of Ghent University, BELSPO (IAP P7/05), the European Union's Horizon 2020 research and innovation programme (consolidator ERC grant agreement No 647755 - DYNPOR (2015-2020)) and the Belgian American Educational Foundation (BAEF). We also acknowledge all current and former CMM members for general discussions on this topic.

\section{References}

[1] C.T. Campbell, J.R.V. Sellers, The Entropies of Adsorbed Molecules, J. Am. Chem. Soc., 134 (2012) 18109-18115.

[2] J.F. Weaver, Entropies of Adsorbed Molecules Exceed Expectations, Science, 339 (2013) 39-40.

[3] K.T. Butler, A. Walsh, A.K. Cheetham, G. Kieslich, Organised chaos: entropy in hybrid inorganicorganic systems and other materials, Chem. Sci., 7 (2016) 6316-6324.

[4] P.G. Yot, Z. Boudene, J. Macia, D. Granier, L. Vanduyfhuys, T. Verstraelen, V. Van Speybroeck, T. Devic, C. Serre, G. Ferey, N. Stock, G. Maurin, Metal-organic frameworks as potential shock absorbers: the case of the highly flexible MIL-53(Al), Chem. Commun., 50 (2014) 9462-9464.

[5] E. Verheyen, L. Joos, C. Martineau, C.J. Dawson, C. Weidenthaler, W. Schmidt, R. Yuan, E. Breynaert, V. Van Speybroeck, M. Waroquier, F. Taulelle, M.M.J. Treacy, J.A. Martensa, C.E.A. Kirschhock, 
Flexibility versus rigidity: what determines the stability of zeolite frameworks? A case study, Mater. Horiz., 1 (2014) 582-587.

[6] R. Gounder, E. Iglesia, The Roles of Entropy and Enthalpy in Stabilizing Ion-Pairs at Transition States in Zeolite Acid Catalysis, Acc. Chem. Res., 45 (2012) 229-238.

[7] A.A. Polyansky, R. Zubac, B. Zagrovic, Estimation of Conformational Entropy in Protein-Ligand Interactions: A Computational Perspective, in: R. Baron (Ed.) Computational Drug Discovery and Design, Humana Press Inc, Totowa, 2012, pp. 327-353.

[8] S. Hikiri, T. Yoshidome, M. Ikeguchi, Computational Methods for Configurational Entropy Using Internal and Cartesian Coordinates, J. Chem. Theory Comput., 12 (2016) 5990-6000.

[9] J.D. Chodera, D.L. Mobley, Entropy-Enthalpy Compensation: Role and Ramifications in Biomolecular Ligand Recognition and Design, in: K.A. Dill (Ed.) Annual Review of Biophysics, Vol 42, Annual Reviews, Palo Alto, 2013, pp. 121-142.

[10] A.J. Jones, E. Iglesia, Kinetic, Spectroscopic, and Theoretical Assessment of Associative and Dissociative Methanol Dehydration Routes in Zeolites, Angew. Chem. Int. Ed., 53 (2014) 12177-12181.

[11] R.Y. Brogaard, R. Henry, Y. Schuurman, A.J. Medford, P.G. Moses, P. Beato, S. Svelle, J.K. Nørskov, U. Olsbye, Methanol-to-hydrocarbons conversion: The alkene methylation pathway, J. Catal., 314 (2014) 159-169.

[12] K. De Wispelaere, S. Bailleul, V. Van Speybroeck, Towards molecular control of elementary reactions in zeolite catalysis by advanced molecular simulations mimicking operating conditions, Catal. Sci. Technol., 6 (2016) 2686-2705.

[13] K. De Wispelaere, B. Ensing, A. Ghysels, E.J. Meijer, V. Van Speybroeck, Complex Reaction Environments and Competing Reaction Mechanisms in Zeolite Catalysis: Insights from Advanced Molecular Dynamics, Chem. Eur. J., 21 (2015) 9385-9396.

[14] K. De Wispelaere, C.S. Wondergem, B. Ensing, K. Hemelsoet, E.J. Meijer, B.M. Weckhuysen, V. Van Speybroeck, J. Ruiz-Martínez, Insight into the Effect of Water on the Methanol-to-Olefins Conversion in H-SAPO-34 from Molecular Simulations and in Situ Microspectroscopy, ACS Catal., 6 (2016) 1991-2002. [15] V. Van Speybroeck, K. Hemelsoet, L. Joos, M. Waroquier, R.G. Bell, C.R.A. Catlow, Advances in theory and their application within the field of zeolite chemistry, Chem. Soc. Rev., 44 (2015) 7044-7111.

[16] V. Van Speybroeck, K. De Wispelaere, J. Van der Mynsbrugge, M. Vandichel, K. Hemelsoet, M. Waroquier, First principle chemical kinetics in zeolites: the methanol-to-olefin process as a case study, Chem. Soc. Rev., 43 (2014) 7326-7357.

[17] L. Benco, T. Bučko, J. Hafner, Dehydrogenation of propane over Zn-MOR. Static and dynamic reaction energy diagram, J. Catal., 277 (2011) 104-116. 
[18] T. Bučko, L. Benco, O. Dubay, C. Dellago, J. Hafner, Mechanism of alkane dehydrogenation catalyzed by acidic zeolites: Ab initio transition path sampling, J. Chem. Phys., 131 (2009) 11.

[19] T. Bučko, L. Benco, J. Hafner, J.G. Angyan, Monomolecular cracking of propane over acidic chabazite: An ab initio molecular dynamics and transition path sampling study, J. Catal., 279 (2011) 220228.

[20] T. Bučko, J. Hafner, The role of spatial constraints and entropy in the adsorption and transformation of hydrocarbons catalyzed by zeolites, J. Catal., 329 (2015) 32-48.

[21] F. Göltl, J. Hafner, Modelling the adsorption of short alkanes in protonated chabazite: The impact of dispersion forces and temperature, Microporous Mesoporous Mater., 166 (2013) 176-184.

[22] S.L.C. Moors, K. De Wispelaere, J. Van der Mynsbrugge, M. Waroquier, V. Van Speybroeck, Molecular Dynamics Kinetic Study on the Zeolite-Catalyzed Benzene Methylation in ZSM-5, ACS Catal., 3 (2013) 2556-2567.

[23] P.M. Zimmerman, D.C. Tranca, J. Gomes, D.S. Lambrecht, M. Head-Gordon, A.T. Bell, Ab Initio Simulations Reveal that Reaction Dynamics Strongly Affect Product Selectivity for the Cracking of Alkanes over H-MFI, J. Am. Chem. Soc., 134 (2012) 19468-19476.

[24] A. Bhan, E. Iglesia, A link between reactivity and local structure in acid catalysis on zeolites, Acc. Chem. Res., 41 (2008) 559-567.

[25] A. Corma, State of the art and future challenges of zeolites as catalysts, J. Catal., 216 (2003) 298-312.

[26] B.A. De Moor, M.F. Reyniers, O.C. Gobin, J.A. Lercher, G.B. Marin, Adsorption of C2-C8 n-Alkanes in Zeolites, J. Phys. Chem. C, 115 (2011) 1204-1219.

[27] F. Eder, J.A. Lercher, On the role of the pore size and tortuosity for sorption of alkanes in molecular sieves, J. Phys. Chem. B, 101 (1997) 1273-1278.

[28] R. Gounder, E. Iglesia, The catalytic diversity of zeolites: confinement and solvation effects within voids of molecular dimensions, Chem. Commun., 49 (2013) 3491-3509.

[29] B. Hunger, M. Heuchel, L.A. Clark, R.Q. Snurr, Characterization of acidic OH groups in zeolites of different types: An interpretation of NH3-TPD results in the light of confinement effects, J. Phys. Chem. B, 106 (2002) 3882-3889.

[30] G. Sastre, A. Corma, The confinement effect in zeolites, J. Mol. Catal. A-Chem., 305 (2009) 3-7.

[31] L. Yang, K. Trafford, O. Kresnawahjuesa, J. Sepa, R.J. Gorte, D. White, An examination of confinement effects in high-silica zeolites, J. Phys. Chem. B, 105 (2001) 1935-1942.

[32] F. Eder, J.A. Lercher, Alkane sorption in molecular sieves: The contribution of ordering, intermolecular interactions, and sorption on Bronsted acid sites, Zeolites, 18 (1997) 75-81.

[33] F. Eder, M. Stockenhuber, J.A. Lercher, Bronsted acid site and pore controlled siting of alkane sorption in acidic molecular sieves, J. Phys. Chem. B, 101 (1997) 5414-5419. 
[34] S. Savitz, F. Siperstein, R.J. Gorte, A.L. Myers, Calorimetric study of adsorption of alkanes in highsilica zeolites, J. Phys. Chem. B, 102 (1998) 6865-6872.

[35] J. Van der Mynsbrugge, J. De Ridder, K. Hemelsoet, M. Waroquier, V. Van Speybroeck, Enthalpy and Entropy Barriers Explain the Effects of Topology on the Kinetics of Zeolite-Catalyzed Reactions, Chem. Eur. J., 19 (2013) 11568-11576.

[36] M. Westgård Erichsen, K. De Wispelaere, K. Hemelsoet, S.L.C. Moors, T. Deconinck, M. Waroquier, S. Svelle, V. Van Speybroeck, U. Olsbye, How zeolitic acid strength and composition alter the reactivity of alkenes and aromatics towards methanol, J. Catal., 328 (2015) 186-196.

[37] D. Teschner, G. Novell-Leruth, R. Farra, A. Knop-Gericke, R. Schlögl, L.s. Szentmiklósi, M.G.l. Hevia, H. Soerijanto, R. Schomäcker, J. Pérez-Ramírez, N.r. López, In situ surface coverage analysis of $\mathrm{RuO} 2$-catalysed $\mathrm{HCl}$ oxidation reveals the entropic origin of compensation in heterogeneous catalysis, Nat Chem, 4 (2012) 739-745.

[38] G.C. Bond, M.A. Keane, H. Kral, J.A. Lercher, Compensation phenomena in heterogeneous catalysis: General principles and a possible explanation, Catal. Rev.-Sci. Eng., 42 (2000) 323-383.

[39] L. Benco, Compensation effect. A DFT study of the activation of $\mathrm{N} 2 \mathrm{O}$ over $\mathrm{M}-\mathrm{CHA}(\mathrm{M}=\mathrm{Fe} 2+, \mathrm{Co} 2+$, RuO2+, RuO+), J. Catal., 298 (2013) 122-129.

[40] T. Bligaard, K. Honkala, A. Logadottir, J.K. Norskov, S. Dahl, C.J.H. Jacobsen, On the compensation effect in heterogeneous catalysis, J. Phys. Chem. B, 107 (2003) 9325-9331.

[41] G.A. Samorjai, Y. Li, Introduction to Surface Chemistry and Catalysis, 2nd Edition, Wiley, New Jersey, 2010.

[42] R. Gounder, E. Iglesia, Catalytic Consequences of Spatial Constraints and Acid Site Location for Monomolecular Alkane Activation on Zeolites, J. Am. Chem. Soc., 131 (2009) 1958-1971.

[43] W.O. Haag, in: J. Weitkamp, H.G. Karge, H. Pfeifer, W. Holderich (Eds.) Zeolites and Related Microporous Materials: State of the Art, 1994, pp. 1375-1394.

[44] B. Smit, T.L.M. Maesen, Molecular simulations of zeolites: Adsorption, diffusion, and shape selectivity, Chem. Rev., 108 (2008) 4125-4184.

[45] F.J.M.M. de Gauw, J. van Grondelle, R.A. van Santen, The intrinsic kinetics of n-hexane hydroisomerization catalyzed by platinum-loaded solid-acid catalysts, J. Catal., 206 (2002) 295-304.

[46] P. Kumar, J.W. Thybaut, S. Svelle, U. Olsbye, G.B. Marin, Single-Event Microkinetics for Methanol to Olefins on H-ZSM-5, Ind. Eng. Chem. Res., 52 (2013) 1491-1507.

[47] C.R.A. Catlow, Prediction of Rate Constants for Catalytic Reactions with Chemical Accuracy, Angew. Chem. Int. Ed., 55 (2016) 9132-9133. 
[48] S. Svelle, C. Tuma, X. Rozanska, T. Kerber, J. Sauer, Quantum Chemical Modeling of ZeoliteCatalyzed Methylation Reactions: Toward Chemical Accuracy for Barriers, J. Am. Chem. Soc., 131 (2009) 816-825.

[49] C. Tuma, J. Sauer, A hybrid MP2/planewave-DFT scheme for large chemical systems: proton jumps in zeolites, Chem. Phys. Lett., 387 (2004) 388-394.

[50] C. Tuma, J. Sauer, Treating dispersion effects in extended systems by hybrid MP2 : DFT calculations - protonation of isobutene in zeolite ferrierite, Phys. Chem. Chem. Phys., 8 (2006) 3955-3965.

[51] C.J. Cramer, Essentials of Computational Chemistry, John Wiley \& Sons Ltd, West Sussex, England, 2004.

[52] Normal mode analyses: theory and applications to biological and chemical systems, Chapman and Hall/CRC, 2005.

[53] J.A. Swisher, N. Hansen, T. Maesen, F.J. Keil, B. Smit, A.T. Bell, Theoretical Simulation of n-Alkane Cracking on Zeolites, J. Phys. Chem. C, 114 (2010) 10229-10239.

[54] S. Svelle, P.A. Ronning, S. Kolboe, Kinetic studies of zeolite-catalyzed methylation reactions 1. Coreaction of [C-12]ethene and [C-13]methanol, J. Catal., 224 (2004) 115-123.

[55] S. Svelle, P.O. Ronning, U. Olsbye, S. Kolboe, Kinetic studies of zeolite-catalyzed methylation reactions. Part 2. Co-reaction of [C-12]propene or [C-12]n-butene and [C-13]methanol, J. Catal., 234 (2005) 385-400.

[56] V. Van Speybroeck, J. Van der Mynsbrugge, M. Vandichel, K. Hemelsoet, D. Lesthaeghe, A. Ghysels, G.B. Marin, M. Waroquier, First Principle Kinetic Studies of Zeolite-Catalyzed Methylation Reactions, J. Am. Chem. Soc., 133 (2011) 888-899.

[57] B.A. De Moor, M.-F. Reyniers, G.B. Marin, Physisorption and chemisorption of alkanes and alkenes in H-FAU: a combined ab initio-statistical thermodynamics study, Phys. Chem. Chem. Phys., 11 (2009) 2939-2958.

[58] S. Grimme, Supramolecular Binding Thermodynamics by Dispersion-Corrected Density Functional Theory, Chem. Eur. J., 18 (2012) 9955-9964.

[59] J.D. Chai, M. Head-Gordon, Long-range corrected hybrid density functionals with damped atom-atom dispersion corrections, Phys. Chem. Chem. Phys., 10 (2008) 6615-6620.

[60] R.F. Ribeiro, A.V. Marenich, C.J. Cramer, D.G. Truhlar, Use of Solution-Phase Vibrational Frequencies in Continuum Models for the Free Energy of Solvation, J. Phys. Chem. B, 115 (2011) 1455614562.

[61] Y. Zhao, D.G. Truhlar, Computational characterization and modeling of buckyball tweezers: density functional study of concave-convex pi center dot center dot center dot pi interactions, Phys. Chem. Chem. Phys., 10 (2008) 2813-2818. 
[62] A. Janda, B. Vlaisavljevich, L.C. Lin, S.M. Sharada, B. Smit, M. Head-Gordon, A.T. Bell, Adsorption Thermodynamics and Intrinsic Activation Parameters for Monomolecular Cracking of n-Alkanes on Bronsted Acid Sites in Zeolites, J. Phys. Chem. C, 119 (2015) 10427-10438.

[63] B.A. De Moor, A. Ghysels, M.F. Reyniers, V. Van Speybroeck, M. Waroquier, G.B. Marin, Normal Mode Analysis in Zeolites: Toward an Efficient Calculation of Adsorption Entropies, J. Chem. Theory Comput., 7 (2011) 1090-1101.

[64] A. Ghysels, V. Van Speybroeck, T. Verstraelen, D. Van Neck, M. Waroquier, Calculating reaction rates with partial Hessians: Validation of the mobile block Hessian approach, J. Chem. Theory Comput., 4 (2008) 614-625.

[65] A. Ghysels, D. Van Neck, V. Van Speybroeck, T. Verstraelen, M. Waroquier, Vibrational modes in partially optimized molecular systems, J. Chem. Phys., 126 (2007) 13.

[66] A. Ghysels, V. Van Speybroeck, E. Pauwels, S. Catak, B.R. Brooks, D. Van Neck, M. Waroquier, Comparative Study of Various Normal Mode Analysis Techniques Based on Partial Hessians, J. Comput. Chem., 31 (2010) 994-1007.

[67] A. Ghysels, T. Verstraelen, K. Hemelsoet, M. Waroquier, V. Van Speybroeck, TAMkin: A Versatile Package for Vibrational Analysis and Chemical Kinetics, J. Chem Inf. Model., 50 (2010) 1736-1750.

[68] J.F. Denayer, G.V. Baron, J.A. Martens, P.A. Jacobs, Chromatographic study of adsorption of nalkanes on zeolites at high temperatures, J. Phys. Chem. B, 102 (1998) 3077-3081.

[69] J.F. Denayer, W. Souverijns, P.A. Jacobs, J.A. Martens, G.V. Baron, High-temperature low-pressure adsorption of branched C-5-C-8 alkanes on zeolite beta, ZSM-5, ZSM-22, zeolite Y, and mordenite, J. Phys. Chem. B, 102 (1998) 4588-4597.

[70] G. Piccini, J. Sauer, Effect of Anharmonicity on Adsorption Thermodynamics, J. Chem. Theory Comput., 10 (2014) 2479-2487.

[71] A. Beste, One-dimensional anharmonic oscillator: Quantum versus classical vibrational partition functions, Chem. Phys. Lett., 493 (2010) 200-205.

[72] N. De Silva, B. Njegic, M.S. Gordon, Anharmonicity of Weakly Bound M+-H-2 Complexes, J. Phys. Chem. A, 115 (2011) 3272-3278.

[73] B. Njegic, M.S. Gordon, Exploring the effect of anharmonicity of molecular vibrations on thermodynamic properties, J. Chem. Phys., 125 (2006) 12.

[74] B. Njegic, M.S. Gordon, Predicting accurate vibrational frequencies for highly anharmonic systems, J. Chem. Phys., 129 (2008) 13.

[75] N. De Silva, B. Njegic, M.S. Gordon, Anharmonicity of Weakly Bound Li+-(H-2)(n) (n=1-3) Complexes, J. Phys. Chem. A, 116 (2012) 12148-12152. 
[76] G. Kresse, J. Furthmüller, Efficient iterative schemes for ab initio total-energy calculations using a plane-wave basis set, Phys. Rev. B: Condens. Matter Mater. Phys., 54 (1996) 11169-11186.

[77] G. Kresse, J. Furthmuller, Efficiency of ab-initio total energy calculations for metals and semiconductors using a plane-wave basis set, Comput. Mater. Sci., 6 (1996) 15-50.

[78] G. Kresse, J. Hafner, Ab initio molecular-dynamics simulation of the liquid-metalamorphoussemiconductor transition in germanium, Phys. Rev. B, 49 (1994) 14251-14269.

[79] G. Kresse, J. Hafner, Ab initio molecular dynamics for liquid metals, Phys. Rev. B, 47 (1993) 558561.

[80] G. Piccini, M. Alessio, J. Sauer, Y.C. Zhi, Y. Liu, R. Kolvenbach, A. Jentys, J.A. Lercher, Accurate Adsorption Thermodynamics of Small Alkanes in Zeolites. Ab initio Theory and Experiment for HChabazite, J. Phys. Chem. C, 119 (2015) 6128-6137.

[81] S.L. Tait, Z. Dohnalek, C.T. Campbell, B.D. Kay, n-Alkanes on $\mathrm{MgO}(100)$. II. Chain length dependence of kinetic desorption parameters for small n-alkanes, J. Chem. Phys., 122 (2005) 13.

[82] G. Piccini, M. Alessio, J. Sauer, Ab initio Calculation of Rate Constants for Molecule-Surface Reactions with Chemical Accuracy, Angew. Chem. Int. Ed., 55 (2016) 5235-5237.

[83] Y.P. Li, A.T. Bell, M. Head-Gordon, Thermodynamics of Anharmonic Systems: Uncoupled Mode Approximations for Molecules, J. Chem. Theory Comput., 12 (2016) 2861-2870.

[84] R. Baron, P.H. Hunenberger, J.A. McCammon, Absolute Single-Molecule Entropies from QuasiHarmonic Analysis of Microsecond Molecular Dynamics: Correction Terms and Convergence Properties, J. Chem. Theory Comput., 5 (2009) 3150-3160.

[85] N. Go, H.A. Scheraga, Analysis of contribution of internal vibrations to statistical weights of equilibrium conformations of macromolecules, J. Chem. Phys., 51 (1969) 4751-\&.

[86] M. Karplus, J.N. Kushick, Method for estimating the configurational entropy of macromolecules, Macromolecules, 14 (1981) 325-332.

[87] R.M. Levy, M. Karplus, J. Kushick, D. Perahia, Evaluation of the configurational entropy for proteins - application to molecular-dynamics simulations of an alpha-helix, Macromolecules, 17 (1984) 1370-1374.

[88] B.J. Killian, J.Y. Kravitz, M.K. Gilson, Extraction of configurational entropy from molecular simulations via an expansion approximation, J. Chem. Phys., 127 (2007) 16.

[89] H. Matsuda, Physical nature of higher-order mutual information: Intrinsic correlations and frustration, Phys. Rev. E, 62 (2000) 3096-3102.

[90] J. Schlitter, Estimation of absolute and relative entropies of macromolecules using the covariancematrix, Chem. Phys. Lett., 215 (1993) 617-621. 
[91] K. Alexopoulos, M.-S. Lee, Y. Liu, Y. Zhi, Y. Liu, M.-F. Reyniers, G.B. Marin, V.-A. Glezakou, R. Rousseau, J.A. Lercher, Anharmonicity and Confinement in Zeolites: Structure, Spectroscopy, and Adsorption Free Energy of Ethanol in H-ZSM-5, J. Phys. Chem. C, 120 (2016) 7172-7182.

[92] R.L. de Sousa, H.W.L. Alves, Ab initio calculation of the dynamical properties of PPP and PPV, Braz. J. Phys., 36 (2006) 501-504.

[93] V.A. Glezakou, R. Rousseau, L.X. Dang, B.P. McGrail, Structure, dynamics and vibrational spectrum of supercritical $\mathrm{CO} 2 / \mathrm{H} 2 \mathrm{O}$ mixtures from ab initio molecular dynamics as a function of water cluster formation, Phys. Chem. Chem. Phys., 12 (2010) 8759-8771.

[94] U.F.T. Ndongmouo, M.S. Lee, R. Rousseau, F. Baletto, S. Scandolo, Finite-temperature effects on the stability and infrared spectra of $\mathrm{HCl}(\mathrm{H} 2 \mathrm{O})(6)$ clusters, J. Phys. Chem. A, 111 (2007) 12810-12815.

[95] M.S. Head, J.A. Given, M.K. Gilson, "Mining minima": Direct computation of conformational free energy, J. Phys. Chem. A, 101 (1997) 1609-1618.

[96] M. Ceriotti, J. Cuny, M. Parrinello, D.E. Manolopoulos, Nuclear quantum effects and hydrogen bond fluctuations in water, Proc. Natl. Acad. Sci. U. S. A., 110 (2013) 15591-15596.

[97] M. Ceriotti, W. Fang, P.G. Kusalik, R.H. McKenzie, A. Michaelides, M.A. Morales, T.E. Markland, Nuclear Quantum Effects in Water and Aqueous Systems: Experiment, Theory, and Current Challenges, Chem. Rev., 116 (2016) 7529-7550.

[98] M. Ceriotti, J. More, D.E. Manolopoulos, i-PI: A Python interface for ab initio path integral molecular dynamics simulations, Comput. Phys. Commun., 185 (2014) 1019-1026.

[99] J. Gomes, M. Head-Gordon, A.T. Bell, Reaction Dynamics of Zeolite-Catalyzed Alkene Methylation by Methanol, J. Phys. Chem. C, 118 (2014) 21409-21419.

[100] D.S. Lambrecht, G.N.I. Clark, T. Head-Gordon, M. Head-Gordon, Simulated Photoelectron Spectra of the Cyanide-Water Anion via Quasiclassical Molecular Dynamics, J. Phys. Chem. A, 115 (2011) 59285935.

[101] E. Ramos-Cordoba, D.S. Lambrecht, M. Head-Gordon, Charge-transfer and the hydrogen bond: Spectroscopic and structural implications from electronic structure calculations, Faraday Discuss., 150 (2011) 345-362.

[102] T. Yamada, M. Aida, Structures of Molecules in Ground and Excited Vibrational States from Quasiclassical Direct ab Initio Molecular Dynamics, J. Phys. Chem. A, 114 (2010) 6273-6283.

[103] P.A. Bash, U.C. Singh, F.K. Brown, R. Langridge, P.A. Kollman, Calculation of the relative change in binding free-energy of a protein-inhibitor complex, Science, 235 (1987) 574-576.

[104] E.A. Carter, G. Ciccotti, J.T. Hynes, R. Kapral, Constrained reaction coordinate dynamics for the simulation of rare events, Chem. Phys. Lett., 156 (1989) 472-477. 
[105] T. Huber, A.E. Torda, W.F. Vangunsteren, Local elevation - a method for improving searching properties of molecular-dynamics simulation, J. Comput.-Aided Mol. Des., 8 (1994) 695-708.

[106] J.R. Gullingsrud, R. Braun, K. Schulten, Reconstructing potentials of mean force through time series analysis of steered molecular dynamics simulations, J. Comput. Phys., 151 (1999) 190-211.

[107] A. Laio, M. Parrinello, Escaping free-energy minima, Proc. Natl. Acad. Sci. U. S. A., 99 (2002) 12562-12566.

[108] L. Rosso, P. Minary, Z.W. Zhu, M.E. Tuckerman, On the use of the adiabatic molecular dynamics technique in the calculation of free energy profiles, J. Chem. Phys., 116 (2002) 4389-4402.

[109] L. Sutto, S. Marsili, F.L. Gervasio, New advances in metadynamics, Wiley Interdiscip. Rev.-Comput. Mol. Sci., 2 (2012) 771-779.

[110] C. Abrams, G. Bussi, Enhanced Sampling in Molecular Dynamics Using Metadynamics, ReplicaExchange, and Temperature-Acceleration, Entropy, 16 (2014) 163-199.

[111] C. Hartmann, R. Banisch, M. Sarich, T. Badowski, C. Schuette, Characterization of Rare Events in Molecular Dynamics, Entropy, 16 (2014) 350-376.

[112] P.G. Bolhuis, D. Chandler, C. Dellago, P.L. Geissler, Transition path sampling: Throwing ropes over rough mountain passes, in the dark, Annu. Rev. Phys. Chem., 53 (2002) 291-318.

[113] I.L.C. Buurmans, B.M. Weckhuysen, Heterogeneities of individual catalyst particles in space and time as monitored by spectroscopy, Nat. Chem., 4 (2012) 873-886.

[114] B.M. Weckhuysen, Chemical Imaging of Spatial Heterogeneities in Catalytic Solids at Different Length and Time Scales, Angew. Chem. Int. Ed., 48 (2009) 4910-4943.

[115] B.M. Weckhuysen, HETEROGENEOUS CATALYSIS Catch me if you can!, Nat. Chem., 1 (2009) 690-692.

[116] M.B.J. Roeffaers, G. De Cremer, J. Libeert, R. Ameloot, P. Dedecker, A.J. Bons, M. Buckins, J.A. Martens, B.F. Sels, D.E. De Vos, J. Hofkens, Super-Resolution Reactivity Mapping of Nanostructured Catalyst Particles, Angew. Chem. Int. Ed., 48 (2009) 9285-9289.

[117] B.M. Weckhuysen, Studying birth, life and death of catalytic solids with operando spectroscopy, Natl. Sci. Rev., 2 (2015) 147-149.

[118] B.M. Weckhuysen, J. Yu, Recent advances in zeolite chemistry and catalysis, Chem. Soc. Rev., 44 (2015) 7022-7024.

[119] Y.V. Kissin, Chemical mechanisms of catalytic cracking over solid acidic catalysts: Alkanes and alkenes, Catal. Rev.-Sci. Eng., 43 (2001) 85-146.

[120] P. Cnudde, K. De Wispelaere, J. Van der Mynsbrugge, M. Waroquier, V. Van Speybroeck, Effect of temperature and branching on the nature and stability of alkene cracking intermediates in H-ZSM-5, J. Catal., 345 (2017) 53-69. 
[121] C. Tuma, T. Kerber, J. Sauer, The tert-Butyl Cation in H-Zeolites: Deprotonation to Isobutene and Conversion into Surface Alkoxides, Angew. Chem. Int. Ed., 49 (2010) 4678-4680.

[122] C. Tuma, J. Sauer, Protonated isobutene in zeolites: tert-butyl cation or alkoxide?, Angew. Chem. Int. Ed., 44 (2005) 4769-4771.

[123] J. Hajek, J. Van der Mynsbrugge, K. De Wispelaere, P. Cnudde, L. Vanduyfhuys, M. Waroquier, V. Van Speybroeck, On the stability and nature of adsorbed pentene in Brønsted acid zeolite H-ZSM-5 at 323 K, J. Catal., 340 (2016) 227-235.

[124] G.M. Torrie, J.P. Valleau, Non-physical sampling distributions in Monte-Carlo free-energy estimation - umbrella sampling, J. Comput. Phys., 23 (1977) 187-199.

[125] J.G. Kirkwood, Statistical Mechanics of Fluid Mixtures, J. Chem. Phys., 3 (1935) 300-313.

[126] R.W. Zwanzig, High-temperature equation of state by a perturbation method. 1. Nonpolar gases, J. Chem. Phys., 22 (1954) 1420-1426.

[127] O. Valsson, P. Tiwary, M. Parrinello, Enhancing Important Fluctuations: Rare Events and Metadynamics from a Conceptual Viewpoint, in: M.A. Johnson, T.J. Martinez (Eds.) Annual Review of Physical Chemistry, Vol 67, Annual Reviews, Palo Alto, 2016, pp. 159-184.

[128] J. Van der Mynsbrugge, S.L.C. Moors, K. De Wispelaere, V. Van Speybroeck, Insight into the Formation and Reactivity of Framework-Bound Methoxide Species in H-ZSM-5 from Static and Dynamic Molecular Simulations, ChemCatChem, 6 (2014) 1906-1918.

[129] A.J. Medford, C. Shi, M.J. Hoffmann, A.C. Lausche, S.R. Fitzgibbon, T. Bligaard, J.K. Norskov, CatMAP: A Software Package for Descriptor-Based Microkinetic Mapping of Catalytic Trends, Catal. Lett., 145 (2015) 794-807.

[130] U. Olsbye, S. Svelle, M. Bjorgen, P. Beato, T.V.W. Janssens, F. Joensen, S. Bordiga, K.P. Lillerud, Conversion of Methanol to Hydrocarbons: How Zeolite Cavity and Pore Size Controls Product Selectivity, Angew. Chem. Int. Ed., 51 (2012) 5810-5831.

[131] D.C. Tranca, N. Hansen, J.A. Swisher, B. Smit, F.J. Keil, Combined Density Functional Theory and Monte Carlo Analysis of Monomolecular Cracking of Light Alkanes Over H-ZSM-5, J. Phys. Chem. C, 116 (2012) 23408-23417.

[132] C. Michel, A. Laio, A. Milet, Tracing the Entropy along a Reactive Pathway: The Energy As a Generalized Reaction Coordinate, J. Chem. Theory Comput., 5 (2009) 2193-2196.

[133] J.F. Dama, G. Rotskoff, M. Parrinello, G.A. Voth, Transition-Tempered Metadynamics: Robust, Convergent Metadynamics via On-the-Fly Transition Barrier Estimation, J. Chem. Theory Comput., 10 (2014) 3626-3633.

[134] O. Valsson, M. Parrinello, Variational Approach to Enhanced Sampling and Free Energy Calculations, Phys. Rev. Lett., 113 (2014) 5. 
[135] P. Tiwary, M. Parrinello, From Metadynamics to Dynamics, Phys. Rev. Lett., 111 (2013) 5.

[136] T.S. van Erp, M. Moqadam, E. Riccardi, A. Lervik, Analyzing complex reaction mechanisms using path sampling, J. Chem. Theory Comput., (2016).

[137] M. Rosa, M. Micciarelli, A. Laio, S. Baroni, Sampling Molecular Conformers in Solution with

Quantum Mechanical Accuracy at a Nearly Molecular-Mechanics Cost, J. Chem. Theory Comput., 12 (2016) 4385-4389.

[138] P.G. Bolhuis, C. Dellago, Practical and conceptual path sampling issues, Eur. Phys. J.-Spec. Top., 224 (2015) 2409-2427.

[139] P. Ferrin, D. Simonetti, S. Kandoi, E. Kunkes, J.A. Dumesic, J.K. Norskov, M. Mavrikakis, Modeling Ethanol Decomposition on Transition Metals: A Combined Application of Scaling and Bronsted-EvansPolanyi Relations, J. Am. Chem. Soc., 131 (2009) 5809-5815.

[140] L.C. Grabow, F. Studt, F. Abild-Pedersen, V. Petzold, J. Kleis, T. Bligaard, J.K. Norskov, DescriptorBased Analysis Applied to HCN Synthesis from NH3 and CH4, Angew. Chem. Int. Ed., 50 (2011) 46014605.

[141] A.A. Latimer, A.R. Kulkarni, H. Aljama, J.H. Montoya, J.S. Yoo, C. Tsai, F. Abild-Pedersen, F. Studt, J.K. Norskov, Understanding trends in C-H bond activation in heterogeneous catalysis, Nat. Mater., 2016, doi: 10.1038/nmat4760.

[142] A.J. Medford, A. Vojvodic, J.S. Hummelshoj, J. Voss, F. Abild-Pedersen, F. Studt, T. Bligaard, A. Nilsson, J.K. Nørskov, From the Sabatier principle to a predictive theory of transition-metal heterogeneous catalysis, J. Catal., 328 (2015) 36-42.

[143] J.K. Nørskov, T. Bligaard, The Catalyst Genome, Angew. Chem. Int. Ed., 52 (2013) 776-777.

[144] Z.W. Ulissi, A.R. Singh, C. Tsai, J.K. Nørskov, Automated Discovery and Construction of Surface Phase Diagrams Using Machine Learning, J. Phys. Chem. Lett., 7 (2016) 3931-3935.

[145] C.M. Wang, R.Y. Brogaard, B.M. Weckhuysen, J.K. Nørskov, F. Studt, Reactivity Descriptor in Solid Acid Catalysis: Predicting Turnover Frequencies for Propene Methylation in Zeotypes, J. Phys. Chem. Lett., 5 (2014) 1516-1521. 